Article

\title{
A User-Centered Mobile Cloud Computing Platform for Improving Knowledge Management in Small-to-Medium Enterprises in the Chilean Construction Industry
}

\author{
Daniela Núñez ${ }^{1,+}$, Ximena Ferrada ${ }^{2,+}$, Andrés Neyem ${ }^{1,+}$, Alfredo Serpell ${ }^{3,+}$ (iD \\ and Marcos Sepúlveda ${ }^{1, *,+}$ iD \\ 1 Computer Science Department, School of Engineering, Pontificia Universidad Católica de Chile, \\ Santiago 7820436, Chile; dsnunez@uc.cl (D.N.); aneyem@ing.puc.cl (A.N.) \\ 2 School of Civil Construction, Pontificia Universidad Católica de Chile, Santiago 7820436, Chile; \\ xferrada@uc.cl \\ 3 Faculty of Engineering, Universidad del Desarrollo, Santiago 7610658, Chile; aserpell@udd.cl \\ * Correspondence: marcos@ing.puc.cl \\ $\dagger$ All authors contributed equally to this work.
}

Received: 9 February 2018; Accepted: 27 March 2018; Published: 28 March 2018

\begin{abstract}
Knowledge management (KM) is a key element for the development of small-to-medium enterprises (SMEs) in the construction industry. This is particularly relevant in Chile, where this industry is composed almost entirely of SMEs. Although various KM system proposals can be found in the literature, they are not suitable for SMEs, due to usability problems, budget constraints, and time and connectivity issues. Mobile Cloud Computing (MCC) systems offer several advantages to construction SMEs, but they have not yet been exploited to address KM needs. Therefore, this research is aimed at the development of a MCC-based KM platform to manage lessons learned in different construction projects of SMEs, through an iterative and user-centered methodology. Usability and quality evaluations of the proposed platform show that MCC is a feasible and attractive option to address the KM issues in SMEs of the Chilean construction industry, since it is possible to consider both technical and usability requirements.
\end{abstract}

Keywords: construction projects; knowledge management; lessons learned systems; mobile cloud computing

\section{Introduction}

The construction industry is a knowledge-based industry [1]. Therefore, effective knowledge management $(\mathrm{KM})$ is believed to be one of the performance enhancers for organizations wanting to remain successful in the highly competitive global construction industry [2]. KM is even more important for small-to-medium enterprises (SMEs), as knowledge is a key resource for them to survive, grow, and remain competitive in turbulent economic times [3]. This has a particular relevance for the Chilean construction sector because $98 \%$ of these construction companies are SMEs and they represent $81 \%$ of the workplaces in this sector [4].

KM recognizes knowledge as one of the most important assets of an organization, moving away from the traditional economic vision, which recognizes knowledge as something external to the company and without connection to the economic process [5]. Knowledge is seen as information that is found in the mind of an individual, related to facts, procedures, concepts, judgments and ideas; in turn, $\mathrm{KM}$ refers to identifying and leveraging collective knowledge in an organization to help the company 
compete [6]. The KM process can be divided into two main macro-processes: knowledge management adoption, and knowledge management development [7]. In this article, we focus mainly in the second macro-process, which includes the phases of creation, storage, transfer, sharing, and application of knowledge [7].

To conduct KM in SMEs, an appropriate environment is needed, where the employees manage their knowledge in their day-to-day activities and do so without it becoming an excessive workload [8]. In order to achieve this in SMEs, Information and Communication Technology (ICT) must play an enabling and even a key role in the KM process [8-10], but most SMEs construction companies lack the budget for investment in ICT [11] or have difficulties adopting ICT [12]. It is difficult for construction practitioners to adopt innovative ICT, even when those technologies have been proven to be beneficial, as technology-centered designs force users to adapt to new technology [13]. Thus, in order to consider ICT tools for KM in SMEs, they need to be inexpensive and user-friendly [8], which can be accomplished with a methodology that emphasizes fast iteration and user insight, such as lean development methodology [14].

The literature has examined programs to conduct KM on Building Information Modeling (BIM)-based platforms [15-17], since they are widely used in several countries. Nevertheless, the usage of BIM for the KM process in SMEs must cope with significant entry barriers, such as high initial investment and staff training [18]. Moreover, BIM is at a very early stage of adoption in the Chilean construction industry, meaning that these barriers will not be surpassed in the medium term $[4,19]$. Thus, there is a need to develop alternative tools that do not depend on BIM to conduct KM in construction SMEs.

Platforms based on Cloud Computing (CC) and/or Mobile Computing (MC) emerge as a possible solution to knowledge management in construction companies. The usage of these technologies offers several advantages to the working environment of SMEs construction companies [20,21]. These technologies can also be integrated in what is known as Mobile Cloud Computing (MCC). This integration results in a synergy that provides the advantages of both CC and MC, where CC mitigates some of MC most important limitations [22,23]. CC and MC have been separately proposed in the literature to address issues within the construction industry, covering a wide span of applications from general-use software to very task-specific applications [21,24-28]. Some MCC systems have also been proposed to improve supply chain management [29,30]. Moreover, different BIM applications such as BIM360 [31], BIMx [32], PlanGrid [33] and A360 [34] are mobile and/or cloud-based. Also, systems to manage knowledge such as Slack [35], XWiki [36], or AnswerHub [37] are available in the market.

Nevertheless, none of the systems mentioned above have simultaneously addressed the need for a MCC-based system aiming specifically to support the KM process in SME construction companies. For example, a MC-based BIM system such as BIM360 uses CC and was designed for the construction industry, but it was not designed to support SME's KM process. Alternatively, mobile and cloud KM systems or collaborative systems, such as XWiki or Slack, were designed to support communication and knowledge sharing among team members, but were not designed to support domain specific issues of KM processes in the construction industry. Therefore, considering the above context, this work focuses on the following research question: "How can a user-centered designed MCC KM platform be developed that is user-friendly, inexpensive, and capable of improving the KM process in SMEs of the Chilean construction industry?"

This research develops a Lessons Learned (LL) platform as a tool to manage knowledge to answer the above question. This article focuses on a user-centered design to develop this platform. To emphasize this approach, the lean development methodology that allows for iterative product development was adopted. This methodology comprises semi-structured interviews that were conducted in three Chilean construction companies, and the design, implementation, and evaluation of the MCC-based platform to support the KM process. 
This research encompasses three main contributions. First, it presents a KM platform that was specifically designed to support the KM process required to improve the on-site execution of construction projects in construction SMEs, promoting a structured dissemination of the knowledge generated in each of the projects developed by the company. For this, the specific characteristics and needs of construction SMEs were taken into consideration, allowing the alignment between the nature of the knowledge generated in these companies, and their operative and technological reality. The use of mobile and collaborative technologies, on a mobile cloud computing environment, makes the KM platform more economical, easy to use, and easy to adopt by construction SMEs. Second, it illustrates how to develop a KM platform using a MCC environment, iteratively and incorporating users. In addition, finally, it describes the validation of the system with real potential users, who used the tool in real environments.

The rest of this article is organized into the following sections. Section 2 focuses on KM and how it has been used in SMEs. Section 3 discusses the requirements of the KM process in construction SMEs based on the literature. We then explain our research methodology based on the lean development process in Section 4. Section 5 introduces the architecture of the KM MCC platform. Next, we present the results of the evaluation of the platform that was made to the end-users and construction experts in Section 6. Finally, we conclude with a summary of the research results and the contributions to the body of knowledge, and make suggestions for future research, in Section 7.

\section{Literature Review}

In this research, we consider Knowledge Management as the process that takes care of identifying and leveraging collective knowledge in an organization to help the company compete [6]. The process of KM can be divided into two main macro-processes: KM adoption, and KM development [7]. The latter includes the phases of creation, storage, transfer, sharing, and application of knowledge [7]. KM has been largely studied for large enterprises. However, some researchers have recently studied the factors that affect KM in supply chains [7], startups [38], and SMEs [7,39,40], identifying the criteria that must be taken into account to develop KM in different scenarios.

Regarding supply chains, different factors affecting the development of KM have been studied, which can be grouped into four main categories: managerial factors, relational factors, environmental factors and socio-political factors [7]. However, these studies have usually left aside human and cultural factors, technical factors (such as information systems and infrastructure) and firm specific factors [7]. In the case of startups, the factors that influence the development of KM can be grouped into seven categories: human and cultural factors, geographical factors, managerial and organizational factors, relational factors, startup specific factors, strategic factors, and technical and technological factors [38]. Since our research focuses on KM in construction SMEs, following we discuss how KM have been addressed in SMEs.

\subsection{Knowledge Management in SMEs}

Although KM has been studied for several years, there has been a tendency to focus on large companies, leaving aside SMEs, despite the importance that this type of organization has for the development of a country [39]. However, recently different researchers have focused on understanding which factors influence the implementation of KM, the singular aspects of the KM process in SMEs, how KM is related to the performance of SMEs, and the development of KM Systems (KMSs) that address SME's specific needs. They are well covered by the following literature reviews: [39] that focuses on the KM process; and $[40,41]$ that focus on KMSs, the factors affecting the spread of KM practices, and the impact of KM on firm's performance.

Studying KM in SMEs is relevant [39] given that there is evidence in the literature regarding the impact that KM has on their economic and financial performance, market performance, technical performance, human performance and organizational performance [41]. In addition, it should be considered that SMEs are not just a replica at scale of a large company [42]. Therefore, in this article 
different aspects of construction SMEs are analyzed in order to specify the requirements for a KM platform that suits their needs.

\subsubsection{Success Factors for the Adoption of KM}

Critical success factors for the adoption of KM in SMEs have been identified. Wong and Aspinwall [43] highlight the following success factors: management leadership and support, culture, strategy and purpose, resources, processes and activities, training and education, human resource management, information technology, motivational aids, organizational infrastructure, and measurement. Meanwhile, Cerchione et al. [41] identify three main categories: human and cultural factors (skill, motivation, training, education, trust and collaboration), technical factors (degree of IT applications, information system, infrastructure) and managerial factors (KM strategy, management style, management leadership, organizational infrastructure, team-work and rewarding).

Durst and Runar Edvardsson [39] state that the areas of KM implementation, KM perception, and knowledge transfer are relatively well researched topics; whereas knowledge identification, knowledge storage/retention, and knowledge usage are poorly understood. In this article, special emphasis is placed on identifying the requirements the own construction professionals have to create, store, transfer and use knowledge; and also on how to encourage construction professionals to share their knowledge, areas that have been seldom studied in construction SMEs.

\subsubsection{Factors that Prevent the Adoption of KM}

Different authors highlight that the factors that prevent the adoption of KM strategies and practices by SMEs are, directly or indirectly, connected to three aspects: knowledge is mainly embedded in human resources; there is a sort of common knowledge, which is shared by all members of the organization; and there is a chronic shortage of human and financial resources [40].

Among the barriers that affect the dissemination of KM practices in SMEs are cultural and financial aspects: return on investment and short-term investments always have priority over long-term ones [40]. In addition to the above, several studies have pointed out that among the main differences between large companies and SMEs regarding KM are that most SMEs do not have explicit KM policies, they work KM at the operational level, they put more emphasis than large companies on management of tacit knowledge, and communication channels are more likely to be between companies rather than internal to the organization [39].

On the other hand, in general SMEs do not have resources dedicated to monitor the evolution of the ICT market, which prevents them from following the technological dynamics [40]. Thus, due to resource constraints and a lack of understanding of ICT-related opportunities, SMEs face difficulties in adopting new technologies [44], which is why they are prone to use old KMSs, such as KMS based on databases and e-mail rather than new KMSs based on cloud computing or crowdsourcing systems [40]. In addition, instead of investing in new KM practices, they prefer to adapt the practices they already know to the new KM requirements [42], where many of these practices are oriented towards management of tacit knowledge [40].

\subsection{Knowledge Management Systems in SMEs}

ICTs are highly relevant to support KM development [42], so the use of KMSs has become a priority for most companies. This is especially important for SMEs, which can obtain greater benefits from the opportunities offered by new ICTs [40].

There are different ways to define KMSs. On the one hand, they can be understood as systems based on information technologies developed to support and improve the organizational processes of creation, storage, transfer, sharing, and application of knowledge [6], or alternatively, a broader definition that states that KMSs include both KM practices (the set of methods and techniques to support the organizational processes of knowledge creation, storage and transfer), and KM tools (specific IT-based systems supporting KM practices) [45,46]. 
Based on the latter definition, SMEs use a wide variety of methods and techniques of KM, according to the nature of the knowledge and/or the KM process [41]. In general, SMEs follow large companies in the development of KM practices [42]. In relation to the KM tools used by SMEs, the literature mentions wikis, web pages, collaborative tools, and management tools, among others [41]. The literature describes that more studies are required in relation to KMSs to support the adoption of KM along the supply chain [7]. In turn, studies show that startups use KM practices, such as class diagrams, use case diagrams, critical knowledge mapping practices, among others, as well as KM tools such as internet forums, enterprise portals, mobile apps, social networks, and collaborative technologies [45].

Regarding supply chains, the alignment between the nature of a company's knowledge and the KMSs used is important, due to three main reasons: (1) a correct alignment can positively affect the KM process; (2) a misalignment can generates problems of ineffectiveness or inefficiency; and (3) in the last twenty years ICT has offered a variety of opportunities in terms of new KM tools that are more economical, easier to use and with an improved performance/price ratio [7]. A similar situation arises when studying KMSs in startups [38].

Unlike large companies, SMEs are highly sensitivity to costs and have lower tolerance to the risk of adopting innovations [47], so they generally look for cheaper systems that also require less training and maintenance. Currently, new trends in ICT have led to the development of new KMSs, creating new opportunities for SMEs, being more economical, friendly and effective systems than traditional ones [42].

This article presents the development of a KM tool, a MCC-based KM platform for construction SMEs, which was designed according to the specific characteristics and requirements of this type of companies, in order to reduce resistance to change, facilitate its implementation, and increase its usefulness as a support tool for on-site management of construction projects, where decision-making based on experience and knowledge validated by the organization is vital to meet the demanding requirements of deadlines and costs that construction projects currently have.

Currently, new trends in information and communication technologies allow the development and deployment of new collaboration systems, such as social media and cloud computing, which are creating new opportunities for both startups and established companies [38]. However, it has not yet extensively investigated how data mining and collaborative technologies, such as cloud computing, are used to improve the efficiency and effectiveness of the different phases of KM development along the entire supply chain of an organization [7]. This article presents the results of the first evaluations of a MCC-based KM platform for construction SMEs that allows to identify the perceptions of users regarding its usefulness as an organizational tool for KM.

\section{Knowledge Management Requirements in Construction SMEs}

This section presents a literature review about the characteristics of the KM process in construction companies and what is needed to conduct it properly, placing special emphasis on the context of Chilean SMEs and mapping each need to a system requirement for a KM platform. Then, recent KM system proposals are reviewed, presenting why they may be suitable for other contexts but not for Chilean SMEs, which explains the research gap addressed by this article. Finally, this section presents MCC as a feasible and realistic option to implement a KM platform that fulfills the requirements of this process within SMEs in the Chilean construction industry.

\subsection{Desired Features of KM Platforms Aimed at Construction SMEs}

$\mathrm{KM}$ is not a new subject within the construction industry. It has been established that it has the potential to significantly reduce fieldwork $[2,48]$ and to save time in construction companies, as it enables the reuse of existing and already tested knowledge to solve problems [49]. In addition, the construction industry has come to learn that, if used in conjunction with KM strategies, ICT tools 
have the capability to positively influence project performance in terms of scheduling and reducing costs as well as improving the performance of quality and safety [50].

Examining KM in the SME context, creating, sharing, using, and protecting knowledge efficiently allows research on new market opportunities and increases in productivity that help to develop a competitive advantage, which consequently leverages into business success [3]. For example, a construction organization's only sustainable advantage lies in its capability to learn faster than its competitors and the rate of change imposed by the external environment [51]. Therefore, there is a need to integrate learning into daily operations and business development [3,42].

In light of this, it is important for a KM platform to allow the live capture and sharing of knowledge, making it possible to capture and reuse knowledge during and after the completion of the project [52]. In order to achieve this, it is important to consider that, by extending KM systems to function on-site, field workers could dramatically increase the number of people inputting into the knowledge repository, so that not only those that attend meetings are included, which also serves to ensure that knowledge is gained directly from the person concerned rather than being received as a sanitized, second-hand version [53]. Thus, a KM platform should support both on-site input knowledge and on-site knowledge reutilization. To this end, it is important to consider how the low speed and instability of the Internet connection can affect a system.

The knowledge and learning generated on a daily basis comes in different forms as well as having different levels of quality and usefulness. Reusable project knowledge often exists as a mixture of tacit and explicit knowledge, rather than as distinctively tacit or explicit knowledge alone [10,54], and, after lessons are collected, it needs to be analyzed and validated before it is disseminated through the organization to guarantee the quality of the information [55]. Therefore, an ICT platform meant for KM work should support ways of sharing, validating, and reusing both types of knowledge.

One way to enhance knowledge sharing, validation, and reuse, is by ensuring readability of the information, meaning that it is expected that tacit knowledge as well as explicit lessons learned in projects is captured and reused in forthcoming projects through the use of a manageable format to manipulate the verified knowledge and the use of a mechanism to capture and disseminate it [56]. Based on interviews with Chilean construction professionals, the authors have proposed a simple LL format for explicit knowledge that is self-explanatory and blog-like [57].

The format of tacit knowledge is notoriously difficult to codify [54] and small group meetings are considered very important for sharing tacit knowledge [58]. Nevertheless, Chilean construction companies lack time during project execution [57], and interactive ICTs are often preferred to face-to face interaction between both co-located and distributed workers since they are non-intrusive and commonly support multitasking [59]. Therefore, a KM ICT platform should foster a non-intrusive manner to share tacit knowledge, which resembles a small group conversation sufficiently well.

As outlined above, one of the main constraints on the acquisition and storage of knowledge in Chilean construction companies is the lack of time during project execution [57]. Therefore, not only the format in which information is shared, but the platform as well should be easy to use. A system with a hard-to-use or overly sophisticated user interface (UI) is discouraging for construction professionals, since they would have to spend a lot of time in order to accomplish what they want to do within the system. In this regard, the features of the platforms can include elaborate data-processing capabilities (such as machine learning) as long as they are available for the user in a simple and straightforward interface.

Another constraint pointed out by Chilean construction professionals regarding knowledge sharing is the lack of KM organizational procedures, meaning that there are no clear indications about what knowledge must be stored, where, by whom, or how [57]. Therefore, a KM platform for SMEs should enable everybody to quickly assume his or her new role in the "game" [3] with clear indications of what is expected of him or her and when he or she must do it. Due to the time constraints mentioned above, the ideal scenario would be one where the system sends an alert (e.g., email or push 
notification) when an action has to be taken, instead of forcing the user to constantly check whether there is something he or she should do.

Finally, features such as on-site input and sending alerts tends to suggest that the KM platform should rely on more than one device, at the very least a server and an on-site client. Besides, it has become very common to see construction workers using their own mobile devices (smartphones, laptops, tablets, among others) while working on the job [60], and with such diversity, the computing capabilities of each one cannot be assured. Thus, it is important to consider that the platform can operate on a wide range of hardware configurations and/or device types. A simple way to achieve this is to have different interfaces of the same platform, which have to be able to easily interoperate with each other so that the company is not forced to buy new hardware or licensing. In line with the lack of a significant ICT budget in construction SMEs [11], this feature is key to limiting costs while achieving something positive and innovative [8].

Based on the literature review presented in this subsection, and the results of interviews with Chilean construction professionals performed by the authors and introduced in [57], the base requirements for a KM platform for Chilean construction SMEs are summarized in Table 1.

Table 1. Construction SMEs' requirements for a KM platform.

\begin{tabular}{ll}
\hline Goal & Requirements \\
\hline Integrate learning into & R1: Live capture and sharing of knowledge \\
daily operations and & R2: On-site knowledge input \\
business development & R3: On-site knowledge reutilization \\
& R4: Being able to function with a low-speed, unstable, or non-existent \\
& Internet connection \\
\hline Achieve knowledge & R5: Identification and usage of different sources of knowledge \\
quality & R6: Validation of knowledge \\
\hline Encourage construction & R7: Readability of collected knowledge \\
professionals to use the & R8: Ease and simplicity of use \\
KM platform despite & R9: Clarity about tasks, timing, and roles \\
their lack of time & R10: Non-intrusiveness \\
\hline Coping with budget & R11: Not being tied to specific or expensive PC hardware \\
constraints & R12: Interoperability at low cost \\
\hline
\end{tabular}

For illustration purposes, and to better understand the operation of a construction SME, following we describe the characteristics of one of the companies studied in this research, which exemplifies the most typical characteristics of this type of company, as well as how they face KM. The company develops several projects at the same time, where each professional in charge of a construction project site manages the generated knowledge independently, because the organization does not have the necessary processes or structure to do so. These professionals are in the field, moving from one project to another. The company does not have an ICT department and cannot allocate too many resources to invest in this area, so the use of these technologies is limited to what is essential for the execution of projects. The computers used are not state-of-the-art and, in many cases, they are not using the latest versions of the software. The company uses e-mail, word processors, spreadsheets, a planning software, some software for drawing plans, as well as a repository to store relevant information about the projects (reports, plans, progress reports, among others). However, the information stored is not organized according to the knowledge and learning achieved, but it is organized by project. The learning that is generated in a construction project site is not transferred to the rest of the projects, so whenever a professional leaves the company, he/she takes the knowledge with him/her.

Hence, the main requirements to manage knowledge in this type of companies include: having a low cost system; easy to use and maintain; that allows access to the knowledge generated in all projects of the company, from any place and at any time; oriented to facilitate the resolution of problems; and 
that recognizes the particularities of the work in the field, in temporary facilities, with a not very stable, or non-existent, Internet connection.

\subsection{Existing KM Platforms and Research Gap in Construction SMEs}

As discussed in the previous subsection, the literature establishes several guidelines for KM platforms for the construction industry, but system implementations have also been proposed. The first implementations [61,62] were Web-based. Most recent academic KM system proposals tend to be BIM-based, even though they use Web technologies. This is consistent with the idea that capturing (and sharing) LL should be done throughout the lifecycle of the project [63] and not only at its end, because BIM may cover the complete lifespan of the construction of a building. For example, developing a BIM-based Knowledge Sharing Management (BIMKSM) system as a Web application [15]. This system uses a single unified database linked to BIM files with different levels of access granted to users based on their roles. KM topics are identified and shared through Computer-Aided Design (CAD) objects in the BIM model. Similarly, another proposed Web-based system (Construction BIM-based Knowledge Management-CBIMKM) [64], which also integrates with a 3D CAD environment could be used. Moreover, general guidelines for developing BIM-based KM systems have been established in a framework [16]. According to it, models should share context information through shared parameters, using Industry Foundation Classes (IFC) files and then knowledge (new ideas and LLs) is associated to a BIM model, which can also be exported in an IFC format.

Nevertheless, the existing BIM-based systems are not the best option for Chilean construction SMEs because they fail to achieve several of the requirements discussed in the previous subsection, as shown in Table 2.

Table 2. Construction SMEs' requirements for a KM platform that are not accomplished by BIM-based systems.

\begin{tabular}{ll}
\hline Requirements not Accomplished & Reason for Failure \\
\hline $\begin{array}{l}\text { R4: Being able to function with a } \\
\text { low-speed, unstable, or non-existent } \\
\text { Internet connection }\end{array}$ & $\begin{array}{l}\text { Knowledge dissemination and real-time access needs a } \\
\text { ubiquitous high-speed broadband Internet }\end{array}$ \\
\hline $\begin{array}{l}\text { R7: Readability of collected } \\
\text { knowledge }\end{array}$ & Time and assistance needed to store or update knowledge \\
\hline R8: Ease and simplicity of use & $\begin{array}{l}\text { High PC requirements to run BIM } \\
\text { Time and assistance needed to store or update knowledge }\end{array}$ \\
\hline $\begin{array}{l}\text { R11: Not being tied to specific or } \\
\text { expensive PC hardware }\end{array}$ & High PC requirements to run BIM \\
\hline R12: Interoperability at low cost & $\begin{array}{l}\text { Upgrading to compatible BIM software versions involves } \\
\text { high licensing costs }\end{array}$ \\
\hline
\end{tabular}

It is vital for the success of BIM that all parties participating in the project use the same programs, the same versions of programs, and IFC standards, which would have to be accomplished before starting the project [65]. BIM is at a very early stage of adoption in Chile [4], meaning that interoperability would be costly, in terms of time, training, licenses, and hardware [18,64], and SMEs may not have sufficient time or budget to accomplish it in order to deal with the urgent matter of KM.

Moreover, budget constraints also hinder the possibility of achieving an acceptable level of usability for KM BIM-based systems, as there are high requirements for PC hardware regarding the use of BIM software [64], meaning that the machines needed to run BIM software properly tend to be more costly than an average computer. Usability in such systems is also hindered by the substantial amounts of time and assistance needed by engineers and managers to use BIM software to edit and update knowledge [64]. 
Besides the budgetary and time constraints, there are other reasons why BIM-based systems are not the best option for KM in this context. Knowledge dissemination and real-time access are important challenges within BIM-based systems [16]. For example, ubiquitous high-speed broadband Internet is essential in order to achieve the requirements of the system [65]. This is an issue for KM as the most effective way for construction personnel to manage information on-site is to retrieve or capture information at the point where they are and at the time when they need it [66], and high-speed broadband Internet access is not guaranteed on-site. Moreover, a high disconnection rate and slow Internet speed are primary barriers to the adoption of BIM-based KM systems by users [64].

Complementing Table 2, another issue to consider is the high dependency on BIM objects. The processes proposed for KM in BIM-based systems show that it is arduous, time-consuming, and/or impossible to store and share knowledge if the BIM models do not exist for the purpose of construction management during the construction phase [64]. This means that knowledge from sources that are not directly mapped into BIM models are difficult to consider in these systems. In Chile, BIM is at a very early stage of adoption. For example, $53 \%$ of respondents to the Chilean National BIM Survey 2016 indicate that they are BIM users, but only $22 \%$ of respondents recognized themselves as regular users [19]. Based on this fact, the high dependency on BIM objects turns into a restriction to use BIM as part of a KM platform in Chile. In fact, it is considered to be a constraint that has an impact on requirements 5,7 and 8 as outlined in Table 1.

Although as it was previously mentioned, the BIM-based systems are an alternative to manage the knowledge of a construction company, and fulfill several of the identified requirements, a company like the one illustrated above (Section 3.1), in general does not have the financial capacity to invest in computer equipment or software licenses with the required capacity to support BIM software, nor to train their staff in these issues, which makes it difficult for them to implement and use BIM technologies to their full capacity.

The fact that these systems fail to fulfill all of the Chilean construction SMEs system requirements for a KM platform leaves open a research gap, which is addressed by the platform proposed in this article through the incorporation of MCC. The system proposed in this article represents an alternative to BIM-based systems to manage the knowledge generated in projects of construction SMEs, by offering a more economical option, using information technologies that allow adjusting the resources used according to the real demand of the company; that does not require special training, being intuitive; which is easy to maintain; and, that does not require high-cost hardware. It is believed that a system like the one proposed could also be of interest for other countries in the same state of BIM implementation as Chile.

\subsection{MCC as an Opportunity for KM in Construction SMEs}

Proposals of ICTs to be used in the construction industry are increasing in number and variety within literature. There are two types of ICTs that stand out among those that are being used and/or proposed for the construction industry: CC $[24,25,27,67]$ and MC $[21,28-30]$. These ICTs may cover a wide span of applications, including several general-use systems [27], project management software [27,28], technologies used in procurement activities [26], and radio frequency identification (RFID) to control materials [29,30], among others. To be considered for KM in SMEs, ICT tools need to be inexpensive and user-friendly. ICTs have attained these features due to their focus on platforms of networks, such as collaborative tools and CC tools, providing people with relationships and teamwork in order to improve the KM process in small businesses [8].

As discussed before, CC has been used in the construction industry for several years now and it has been effectively demonstrated to ease development of construction-related architectures [27]. Nevertheless, developing cloud-enabled software applications for mobile environments is considered a challenging task because of the difficulties associated with synchronizing the mobile frontend with the cloud backend while maintaining a minimum set of quality of service requirements that a user would expect in commercial applications [68]. Thus, effective mobile construction solutions are required to 
take advantage of the cloud to perform extensive computations and to store data beyond the intrinsic capabilities of mobile devices. Infrastructures that allow mobile platforms to harness the resources of the cloud and support the aforementioned features that belong in the category of Mobile Cloud Computing (MCC) [68].

MCC architecture consists of a mobile and cloud component connected by the Internet through a mobile network. MCC enabled clients may then use these resources to either increase processing speeds, reduce battery consumption, enable previously unavailable features or reduce mobile network data traffic to reduce costs (the latter is only possible when using nearby short-range wireless enabled clusters). Cloud services in MCC architectures are deployed on a cluster of servers usually managed by a cloud service provider (such as Amazon Web Services or Microsoft Azure). In this approach, we identify three main classifications of cloud computing services, according to the abstraction level of the capability provided and the service model of providers: Infrastructure as a Service (IaaS), Platform as a Service (PaaS), and Software as a Service (SaaS). IaaS is highly customizable because developers are responsible for everything from setting up the operating system to deploying the application and configuring its runtime environment. General PaaS solutions like Web and Worker do a little more of the heavy lifting for developers because the service comes with an Operating System (OS) and certain middleware applications already configured. A subset of PaaS solutions targeted towards particular applications, such as web, media, or mobile, could supply even more of the glue code that the application needs. Finally, SaaS allows for little to no customization and supports only a specific software distribution. As such, SaaS solutions are tailored towards a very particular set of requirements.

In order to ease the integration of cloud services and mobile clients, the concept of cloud Backend as a Service (BaaS) emerges as a solution that allows developers to establish complex mobile-cloud interactions with very little configuration. This kind of software solution provides developers with two major features: on the client side, we have custom libraries for mobile clients made specifically for each relevant mobile operating system; on the server side, we have control panels that make extensive configuration possible in a matter of minutes. BaaS enables not only the connectivity and scalability that comes with all cloud-based services, but also supplies solutions for common mobile development challenges like user authentication, push notifications, data storage, social media integration, geospatial queries, offline sync, analytics, machine learning techniques and more. This provides a consistent way to manage mobile backend requirements as services and removes the need for having to develop custom ad-hoc solutions that often suffer from serious performance and security issues

In the previous paragraphs we explained how Mobile and Cloud Computing represent a drastically different approach to IT solution delivery and how this technology could be one of the foundations of the next generation of computing [68]. Therefore, a MCC-based platform for KM in the construction sector could take advantage of the benefits of both connectivity and scaling. Moreover, MC and CC paradigms intensify and increase the effectiveness of each other, as MCC makes it possible to address many of the benefits that contractors seek using the combination of these two technologies, such as ubiquitous access, scalability, reduced need for in-house IT, and collaboration opportunities [69]. These benefits are in line with the already reviewed requirements of a KM platform and help to overcome the limitations mentioned for the existing systems. Therefore, MCC represents a good opportunity to implement a suitable and effective KM platform for construction SMEs [70]. This opportunity has not been adequately exploited so far in knowledge management applications specifically developed for construction companies.

\section{Materials and Methods}

To solve the issues identified in the previous section, we envision a platform that can store and distribute knowledge gained from on-site experience, sharing data in real-time between different field teams within the same construction company and its head office. This section describes a user-centered approach that could be implemented to achieve this. 
The KM platform was developed using a Lean development methodology. This methodology is based on cycles, each of which is composed of three distinctive phases (Build, Measure and Learn). The characteristics of this methodology are described in Section 4.1. Three cycles were developed, which are described in Section 4.2. Section 4.3 describes how the empirical evaluation of the KM platform was carried out while Section 6 describes in detail the results of such empirical evaluation.

\subsection{Research and Development Method}

One of the most important aspects of this research is to continuously include input from the potential end users of the proposed platform in order to achieve true user-friendliness. Systems described in previous sections have not completely succeeded in this point because of their complexity or their lack of understanding of some key requirements, such as the ability of working with an unstable Internet connection. To build a cloud platform that properly addresses the requirements stated in previous sections, we relied on User-Centered Design (UCD) principles. A UCD process requires the understanding of the target users, their tasks and environments. It incorporates the user feedback by directly involving them during the design and development. UCD principles have been used successfully together with agile development processes [71-73].

To further emphasize the user-centered approach, lean development methodologies [14] were adopted to identify, satisfy and test the requirements of the platform. At its core, lean development methodology is based on Lean Startup principles [74] and it focuses on formulating a hypothesis and then quickly testing the assumptions surrounding it. It achieves this by establishing an iterative development process intent on building a Minimum Viable Product (MVP) around that hypothesis as quickly as possible. By working in concrete MVPs we will learn about the crucial design decisions and trade-offs that impact the final behavior of the KM platform. Moreover, the lean philosophy makes it possible to minimize the company's costs, eliminating any and all types of waste, and to increase profitability, giving more value to the products and services developed [75]. Then, the usage of this methodology is consistent with the context being worked on in Chile, since among the main barriers to the entry of ICTs in construction SMEs are the issues of small ICT investments [11] and worker's skepticism about the value they bring [76].

In this methodology, every iteration defines a research cycle divided into three distinct phases (Build, Measure and Learn) in which many people have professional training that focuses on one single element of this feedback loop (shown in Figure 1). Due to this, by using this approach it allows this research to easily and properly integrate multidisciplinary perspectives to propose a solution for $\mathrm{KM}$ in construction companies. The formation of an interdisciplinary team is particularly relevant as construction experts and professionals have greater insights into the construction industry's needs, while key factors for the success of mobile services are identifying and investigating how users are influenced and then how they behave, and finally revealing what they really expect (i.e., needs and preferences) [77].

Details of the Build-Measure-Learn cycles performed by this research are described in Section 4.2. Later, details of the empirical evaluation performed in the Measure phase of the second and third iterations are described in the Section 4.3.

\subsection{Research Cycles}

This research has completed the Build-Measure-Learn cycle three times so far. The second and third cycles were carried out normally, but the first one was slightly adapted to have an initial and final Learn phase due to the existence of previous research regarding ICT systems that support the $\mathrm{KM}$ process in construction companies. The main activities for each phase of each iteration are shown in Figure 1. These activities include building a system proposal using the available knowledge and information about the problem at the time of initiating the loop (Build phase), then, evaluating it, for example, through ISO Quality Systems and Software (QSS) or System Usability Scale (SUS) 
questionnaires (Measure phase), and finally, analyzing the results, generating new insights that serve as input for the next iteration (Learn phase).

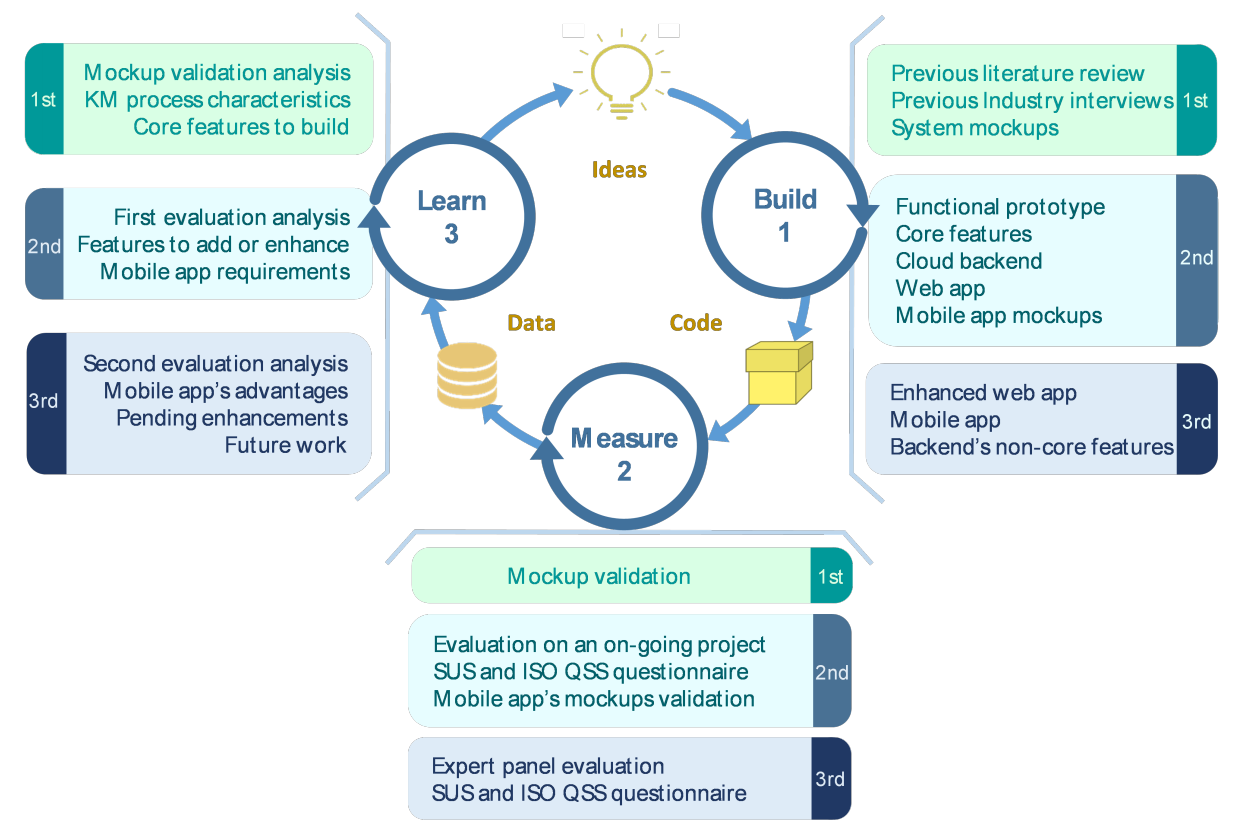

Figure 1. The Build-Measure-Learn feedback loop and the main activities performed on the first, second, and third cycles.

\subsubsection{First Cycle}

During the initial Learn phase, we identified deficiencies in the KM and LL processes through a literature review and semi-structured interviews with 16 construction professionals from three different construction companies. The object of these interviews was to understand first, how these construction companies currently manage their knowledge; second, what the main barriers for knowledge management are; and finally, how a lessons-learned platform for construction professionals should be to be useful in their day to day activities. In total, the professionals interviewed comprised eight project managers, five project supervisors, two quality managers, and one chief operating officer (COO). Regarding the two first objectives [57], construction professionals recognized the lack of organizational procedures to manage knowledge. They indicated that they do not store knowledge because it is not clearly defined what information or knowledge they need to store, what format to store it in, or where to store it. Also, interviewees indicated that communication and cooperation between professionals of the same company is difficult and slow, given the geographical location of projects. Finally, they mention the lack of time during the execution of projects as a major limitation to capture and store knowledge.

During the Build phase, initial mock-ups were made. Later, the Measure phase consisted of new semi-structured interviews with construction experts within the companies to evaluate the initial platform mock-ups. A total of one chief executive officer (CEO), one COO, two project supervisors, and one quality manager participated in these later interviews, letting the researchers know which features added more value to their company's work. Finally, in the second Learn phase, those comments and feedback were analyzed and consolidated along with the literature review, resulting in an understanding of the KM process as a challenge with specific characteristics in the SMEs' context, as presented in Section 3 of this article. 


\subsubsection{Second Cycle}

The goal of this cycle was to build a functional prototype using the mock-ups and requirements resulting from the first cycle, in order to see how the platform is perceived by on-site professionals. In order to do so, during the Building phase, the core functionalities were implemented in a cloud service. A simple Web application to access this service was built as well, since a Web application can be accessed from a mobile device and / or a desktop device, allowing the exploration of both kinds of user experiences with minimum development effort. During the Measure phase, we worked in depth with one company from the previous cycle. A team of on-site professionals used the prototype to register and access LL from an on-going project and then participated in a focus group, which allowed us to learn their opinions on the prototype and their expectations about the platform through a three-part questionnaire. Section 4.3 explains each part of the questionnaire in more detail, while its results are presented in Section 6.1. In addition, the periodic interviews continued, this time with the COO and Innovation Manager, in order to consider both the operational and the managerial vision. Finally, during the Learn phase, the results and comments from the questionnaire, and the interviews with the managers were analyzed in order to first evaluate the relevance of using mobile and cloud technologies for the construction industry and secondly extract functional and non-functional requirements for the next iteration of the development of the platform. The main results of this cycle are presented and discussed in Section 6.1.

\subsubsection{Third Cycle}

This cycle focused on further development of the core functionalities as services and the mobile applications to consume them, as well as on a wider evaluation including construction professionals with different profiles and backgrounds. The Build phase focused on the development of a cross-platform mobile application and the improvement of the existing cloud service, following the guidelines obtained in the previous cycle. This is presented in detail in Section 5.3. During the Measure phase, expert construction professionals were consulted and interviewed, in order to keep in mind both the operational and the managerial vision, while at the same time widening the range of respondent's ages and technological backgrounds. These results are presented in Section 6.2.

\subsection{Empirical Evaluation for the Measure Phase}

Once there was an implementation of at least a prototype of the platform (beyond mock-ups), a three-part questionnaire was created to evaluate the capacity of the platform as a tool for the improvement of the KM process in Chilean SMEs. This test evaluated mainly usability and usefulness factors such as timely access to information, structure, and ease of the KM process within the system, as well as consistency with everyday work.

One part focused completely on the usability assessment, according to the SUS test, which defines 10 questions that are evaluated on a Likert scale of 1 to 5 [78]. A SUS score between 70 and 80 is considered "good", between 80 and 90 is considered "excellent", and over 90 is considered the "best imaginable" [79]. According to the literature, obtaining a SUS score at each step in an iterative development process allows the experimenter to compare each iteration using a standard tool [80]. Besides, considering the issue of the lack of time present in Chilean construction SMEs as discussed earlier, SUS presents a powerful advantage: SUS makes it possible to get a measure of the perceived usability of a system with a small sample (a total of 8-12 users), resulting in a fairly precise assessment of how people see the system or product [81].

To complement the SUS test, the second part of the test took into account an overall assessment of the platform, considering elements defined in the ISO/IEC 25010: 2011 quality software standard [82], as well as other elements considered relevant to the project. This part consisted of 11 affirmations about the platform as show in Table 3 which had to be ranked from 1 (totally disagree-worst) to 5 (totally agree- - best) to know if the respondent agreed to these statements or not. Considering the 
additive nature of this ranking, each evaluator could assign a minimum score of 11 (each affirmation evaluated as 1) and a maximum score of 55 to the system. Considering the ratio between the total score (TS) and the number of affirmations (QA), one can interpret each professional's evaluation using a scale from 1 to 5 [80].

The last part consisted of open questions, in order to identify in greater detail the perceptions and opinions of users according to their professional experience in the construction sector.

Table 3. Affirmations to answer using a Likert scale regarding the propose platform.

\begin{tabular}{l}
\hline Question \\
1. The system responds to my requests in a timely manner \\
2. The system can be used in different environments, for example, at central office and on site \\
3. The system prevents access by persons outside the company or without associated permissions \\
4. The system offers a structured way of storing knowledge in the company \\
5. The system provides a useful way of storing knowledge in the company \\
6. The system allows quick access to the information and knowledge stored in the company \\
7. The system will increase the amount of stored knowledge in the company \\
8. The system will increase the amount of knowledge available to professionals in the company \\
9. The system will allow the active participation of company's professionals in the lessons learned process \\
10. The knowledge stored in the system will improve the process of decision making in the company's projects \\
11. The system is suitable for use in everyday work
\end{tabular}

\section{The Proposed KM MCC Platform}

Integrating the literature review and the input from the construction professionals, a general overview of the expected use of the platform in the construction SME work scenario is shown in Figure 2. For instance, it is known that in a construction project, new knowledge can be created every day. Then, during the execution of daily activities, an on-site professional can identify something his or her team has learned that would most probably be useful for another project within the same company. This professional can enter this knowledge in the platform in the form of an LL, using the device of his or her preference and attaching multimedia if necessary. If there is no Internet connection, the information is saved on the device to be uploaded later. Once the professional has finished his or her LL draft, the project manager (or someone appointed by him or her) has to verify the content of the LL and ask for corrections or approve it. Another option would be for head office experts or supervisors to participate in a second verification process, strengthening the quality of the stored knowledge. This second verification is not mandatory because if it were it could create a bottleneck. Once the LL has passed the first verification process, it becomes available to the rest of the company and can be accessed through direct search or cloud-based lessons recommendations, allowing knowledge reutilization. These professionals are probably elsewhere in the company or even the country. People who read and use the LL available can give feedback about its usefulness and complement it through comments. This information is later used by users to learn how to enhance the content they share. It is used by the platform, as well, to produce better suggestions and improve the search results for each user.

\subsection{The Architecture of the KM Platform}

In order to satisfy the requirements discussed in the literature review and the expected usage, the proposed platform architecture consists of a series of software and hardware layers. The KM platform was built with the facilities provided by Microsoft Azure. This cloud vendor was selected to support this platform because it fits its current status and future plans. Having a KM platform hosted using Azure cloud services provides a flexible and extensible system for performing different data processing tasks. This platform can be dynamically scaled to fulfill the several computational requirements in a cost-efficient way, such as processing heavy tasks or managing complex data from different sources. 


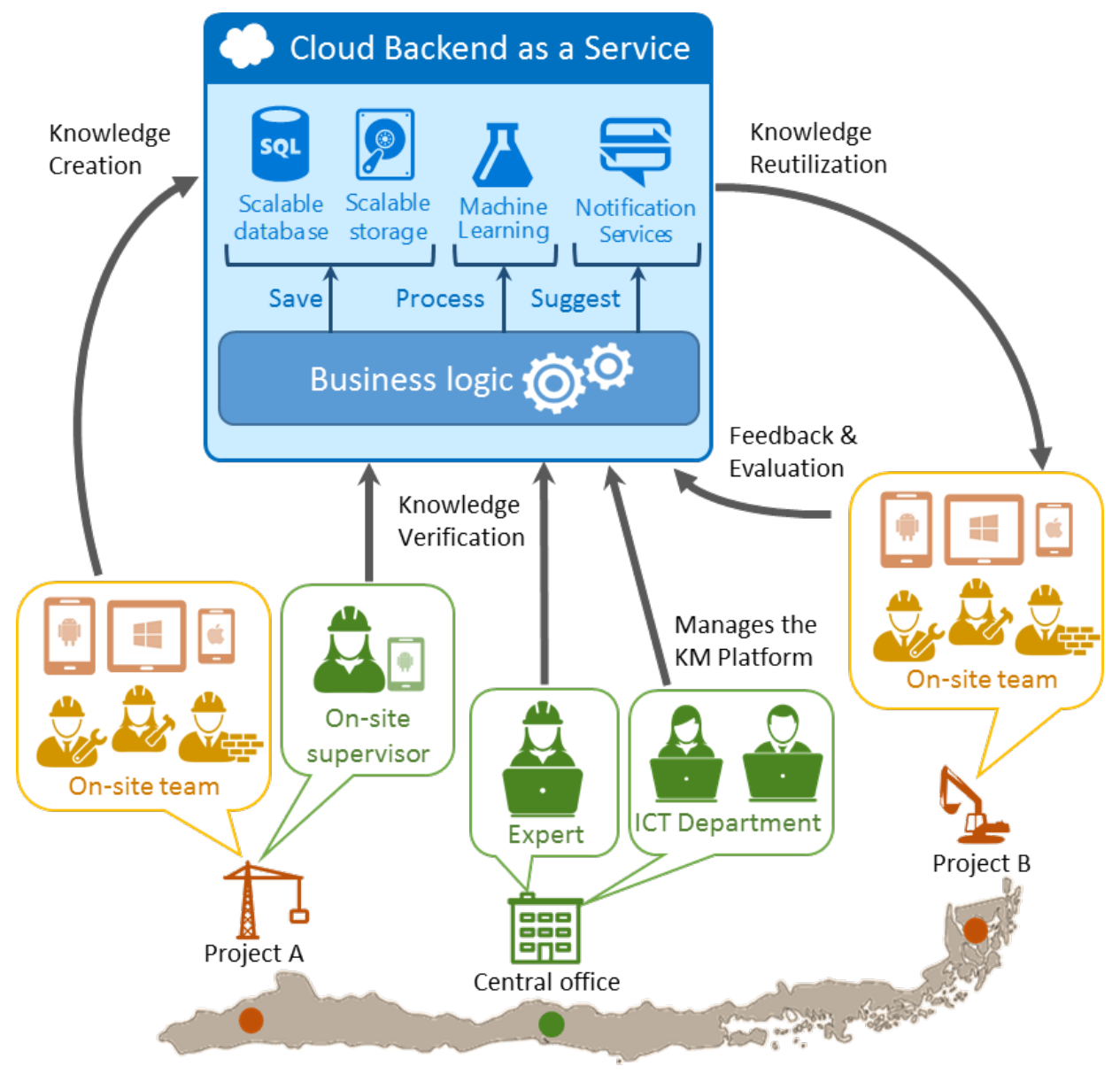

Figure 2. General overview of the use of the KM platform.

The interrelationships between the different layers are shown in Figure 3. Next, a description of each layer and its role in the fulfillment of requirements is presented.

- Mobile UI: Cross-platform mobile application to be installed on the devices of on-site professionals. It is associated with requirements R1, R2, R3, R4, R5, R6, R7, R8, R9, and R10.

- Web UI: Web application that is accessible from any device with a Web browser. It is associated with requirements R1, R5, R6, R7, R8, R9, and R10.

- Cloud Backend as a Service: Application Programming Interfaces (APIs) that expose the functionalities of the application logic to the different UIs, adapting the requests and responses to the most suitable format and content for each of them. It is associated with requirements R4, R12, and R11.

- Application Logic: Centralized components that handle and implement the business logic, maintaining the consistency of the information regardless of which client accesses it. It is associated with requirements R3, R5, R6, R8, R9, and R10.

- Domain Models: Abstractions that represent the information units to be stored and/or processed (e.g., a lesson, a project, a microblog message, among others) and how they are related to each other. Are associated with requirements R5, R6 and R7.

- Cloud PaaS: Software components that access the services provided by the cloud vendor, in the form of a platform as a service. As a different cloud provider could be used in the future, it defines interfaces to be used by the rest of the application, providing a concrete implementation that depends on the cloud provider contracted at present. It is associated with requirements R11 and R12. 


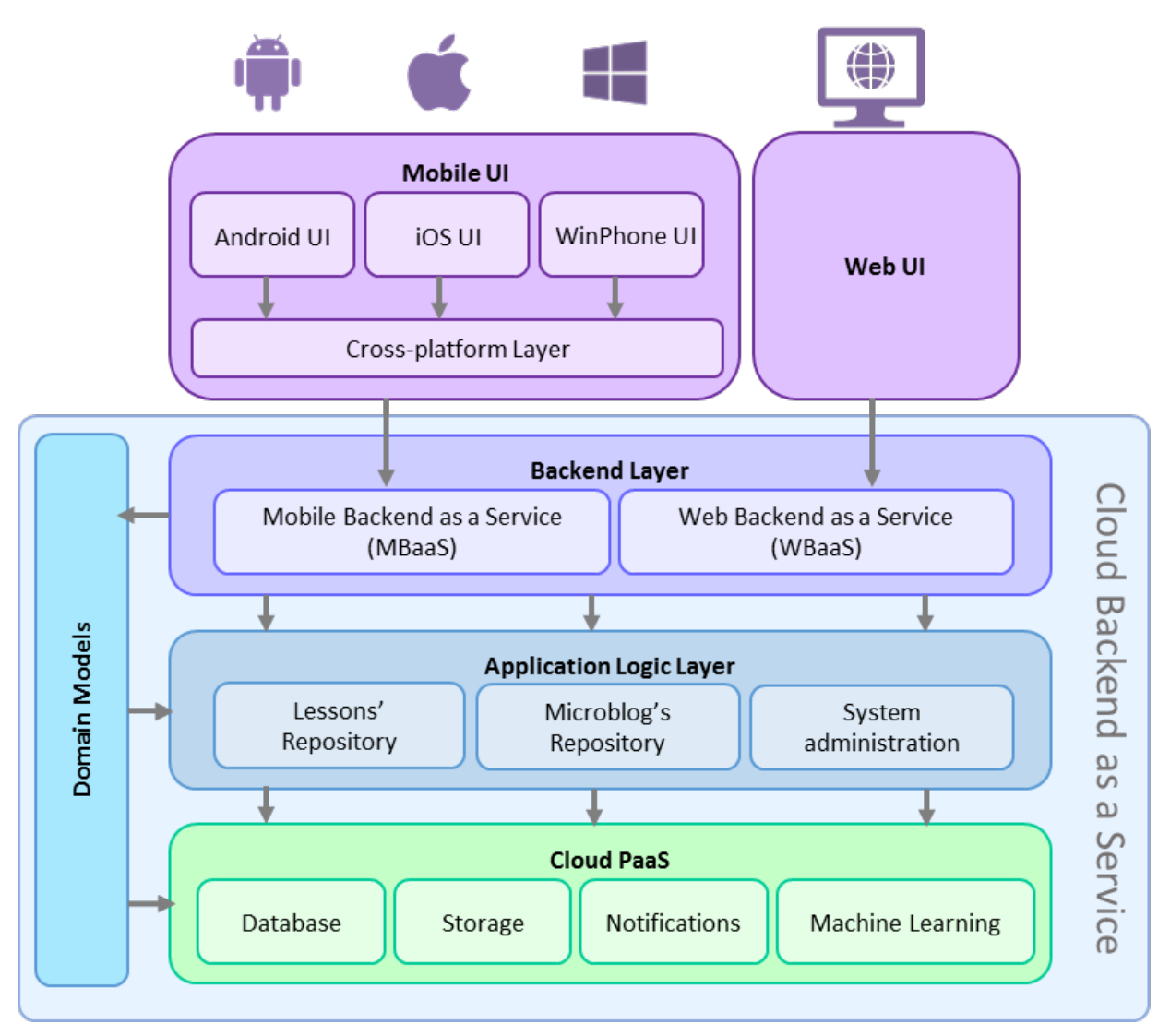

Figure 3. Proposed architecture for the MCC-based KM platform.

The most widely known layers are the Web application, mobile application, and cloud Backend as a Service (BaaS), as they encompass the complete architecture. Thus, the following subsections explain the functionalities of the platform based on these three layers.

\subsection{Web Application}

The first version of the Web application was the result of the second Build-Measure-Learn cycle and was enhanced during the third cycle. As an example of the final version of this application, Figure 4 shows a screenshot from the LL form. The implementation of this version is based on ASP.NET MVC because it provides a reliable framework for web development that integrates easily with different databases via Entity Framework. ASP.NET was also chosen because it includes the Web API framework. Given the BaaS software architecture pattern used in KM platform, Web API was needed to easily create and deploy an API to access the application. Finally, this Web application version laid the foundation of the functionality of the entire platform, which mainly consists of a microblogging platform and an LL database manager. These modules are explained next. 


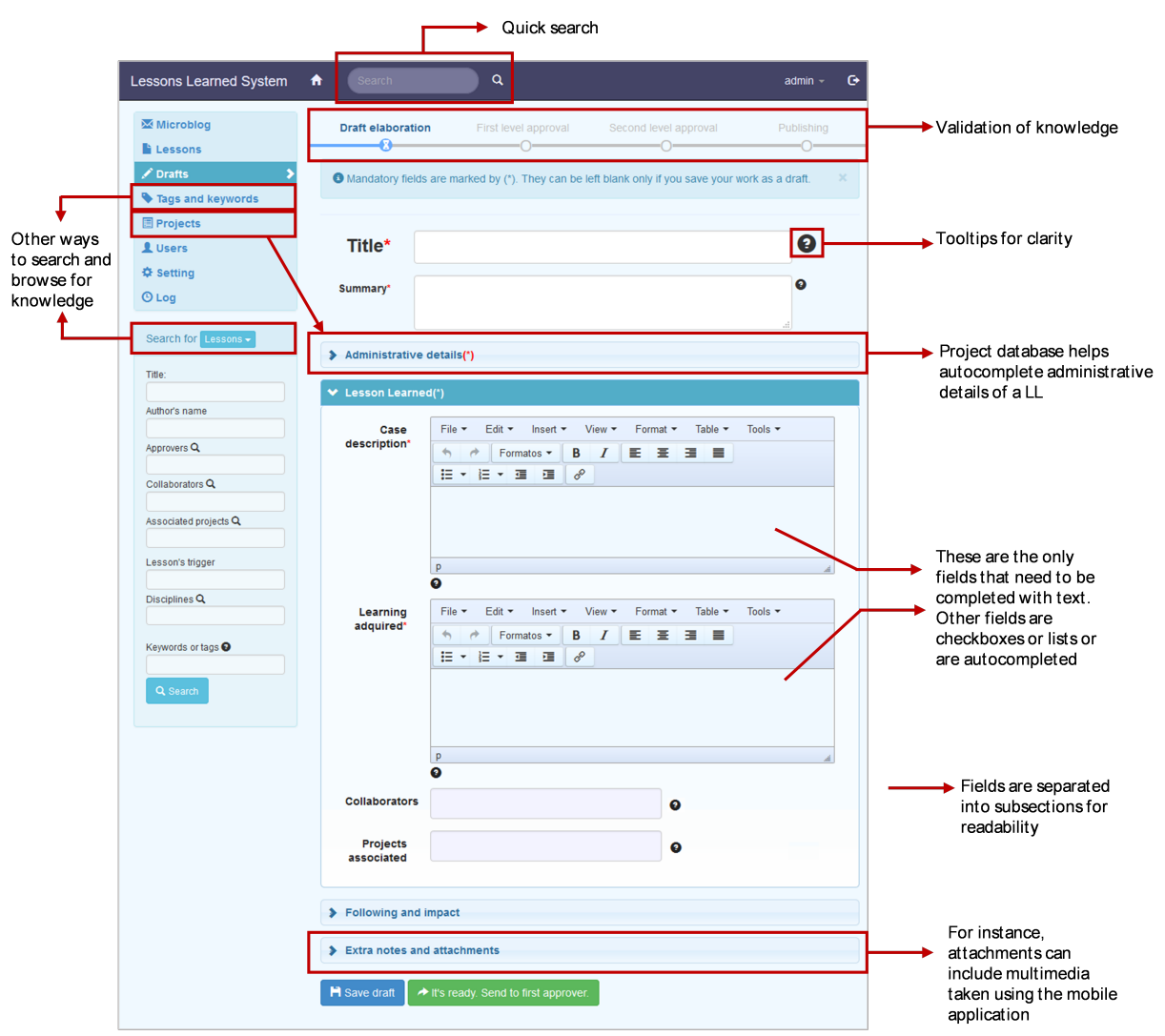

Figure 4. Screenshot from the LL form in the Web application.

\subsubsection{Microblogging Platform}

This functionality aims to cope primarily with R5 and R10 (but also with R1 and R2). The organizational microblog is designed to allow a more fluid contact among professionals, fostering tacit knowledge transfer. The primary objective of the microblog is the dissemination of tacit knowledge that may be relevant to other users via short, timely messages emulating face-to-face formal and informal communications and conversations, thereby allowing these interactions to be distributed without the use of fixed time intervals, thus overcoming the lack of available time and geographical dispersion.

In order to foster the transfer of tacit knowledge, conversations should be not only stored in the system but also made public if the users allow it. In this way, anyone can consult them and/or participate, adding their own experience into the mix. The microblogging platform fulfills the following requirements:

- Users have a personalized view in which they see the last public messages and the ones directly addressed to them.

- Messages can be addressed to all community members or to specific groups of users.

- Messages addressed to specific groups of users or individual users can be marked as private or public.

- Relevant messages for a particular user are highlighted. Their relevance is determined by three factors: whether or not it is directly addressed to a particular user, his or her role in the organization, and previous collaboration with the message's author.

\subsubsection{LL Database}

This is the core of the platform. The LL database uses BLOB storage to save multimedia from LL and uses SQL Azure databases to save these records. Thus, the LL database allows the storage, 
reuse, and transfer of knowledge created in the design and construction phases of a project as a formal process, avoiding the recurrence of mistakes. This system recognizes four types of users: a lesson creator, an approver, a consultant and a system manager, in order to achieve R8 and R9. The platform supports four main processes performed by these users on the database, as follows:

\section{(a) Lesson creation}

As LL are the intellectual assets used to create value based on past experience [76], project managers must decide what project experiences have strategic or operational relevance and then ask a professional with the role of lesson creator to write the new lesson using the format defined by the authors [57]. Input from users showed that they were familiar with blog-like systems, so an interface of similar characteristics was chosen (consistently with R7 and R8), especially considering that the lack of time is an important constraint on storing knowledge (writing was maintained at the minimum required level, incorporating auto-completion and checkboxes, photos, videos, and audio when possible). Once the user has completed the LL form (for instance, using the web interface shown in Figure 4), he or she must send the newly created lesson for approval. As the context of the lesson is important for its later use, information regarding the project where the lesson was created is included, such as the project name and address, approvers, construction volume, price, project type, contract type, client type, starting date, duration, and end date.

\section{(b) Lesson approval (R6 and R9)}

Each new lesson has to be verified and approved by one or more approver users according to the process defined by each company. The platforms built-in process is shown in Figure 5. Lesson creators are typically on-site professionals and the lesson approver should be the project manager or someone designated by him/her. This process allows the review and improvement (if required) of a lesson. Also, it gives accountability and more credibility to the lessons included in the database and clarity about the roles and responsibilities of each actor in the KM process, in terms of the actions required and the time in which they should be performed (R9).

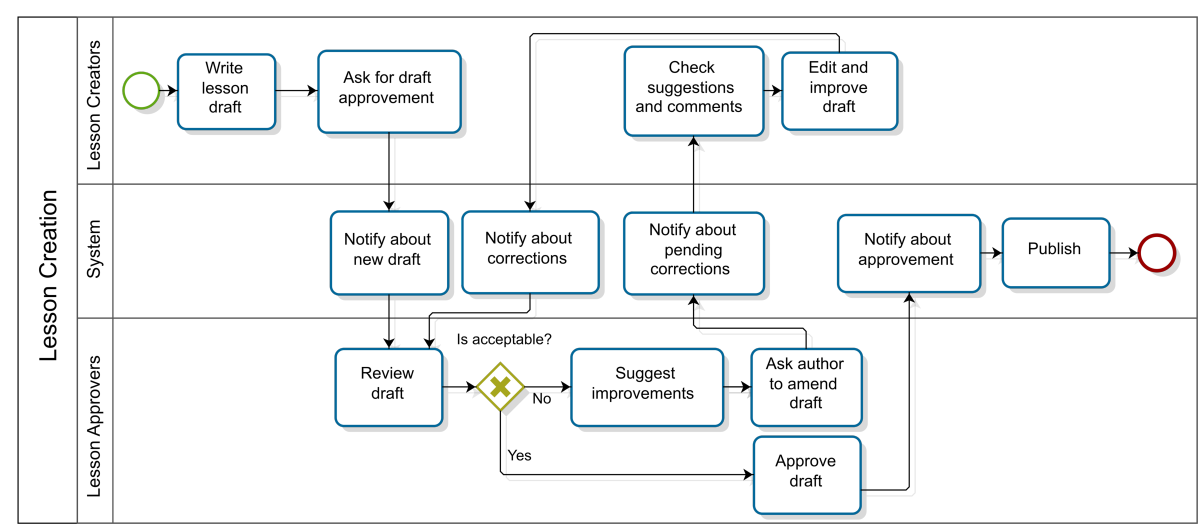

Figure 5. Proposal for the approval process for lessons learned.

In order to alert the users when they have to perform an action regarding this process, the platform sends them push notifications when appropriate. Only when a lesson completes this process without any comments is it published (R6). Optionally, and depending on the time available, the LL can receive a second seal of approval, but this time not from the project manager but from a supervisor or expert from head office. In both the first and second approvals, the lesson approver could send comments to the lesson creator with modifications that need to be made before the lesson is published, or they could just approve the lesson, as shown in Figure 6. 


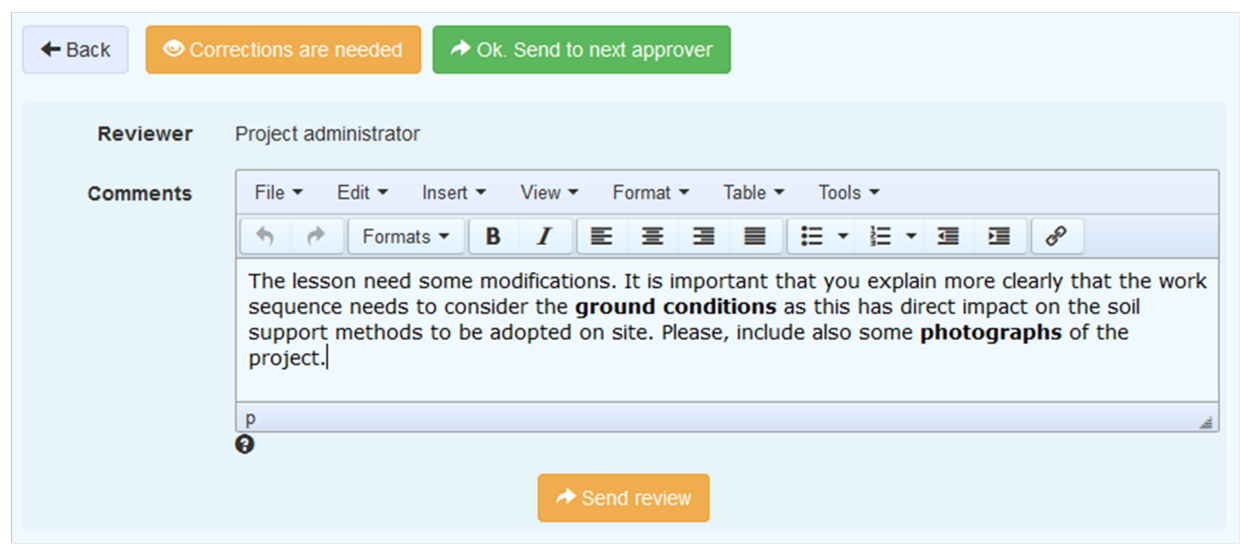

Figure 6. Screenshot of the web application showing the review option.

(c) Lesson search (R8)

It is possible to search lessons using a quick search feature, which allows searching by keywords, and using an advanced search, which allows searching using filters such as lesson title, author, approvers, project name, disciplines, source of the lessons, and lesson tags.

(d) Lesson evaluation (R6) and future recommendations (R8)

Once a user has found a lesson in the database, he or she can post a comment regarding his or her experience using this lesson, and/or express how useful the lesson is for his or her work through an evaluation system on a scale from 1 to 5 , with 5 indicating the highest usefulness. What is evaluated is the "usefulness" instead of the "quality", because, during the semi-structured interviews performed to evaluate the first functional prototype (during the second research cycle explained in Section 4.2) SME construction professionals stated that the evaluation of quality would be perceived as aggressive, discouraging people from sharing their knowledge, as they would fear receiving poor evaluations.

The latest user evaluation showed that a search system is necessary but not sufficient to fulfill $\mathrm{R} 8$, as users still have to make time to search for useful knowledge. They said that the ideal scenario for them would be if the system told them, in advance, what they should be reading. In this regard, content-based recommender systems provide recommendations to users based on comparing items or products to the items in which the user has shown an interest [83], and collaborative filtering technology, as well as other forms of recommendation, automates the process of sharing opinions on the relevance and quality of information [84]. The advanced capabilities of MCC make it possible to apply machine learning algorithms to the information being generated by the feedback and evaluations, allowing the generation of suggestions and recommendations. Therefore, we enhanced the proposal through the incorporation of a content-based recommender component as a part of the cloud backend service. Figure 7 shows the current version of the front-end web application of this solution running on the cloud. This follows the guidelines of $\mathrm{R} 8$, as this automation reduces the time that professionals must spend searching for relevant information. 


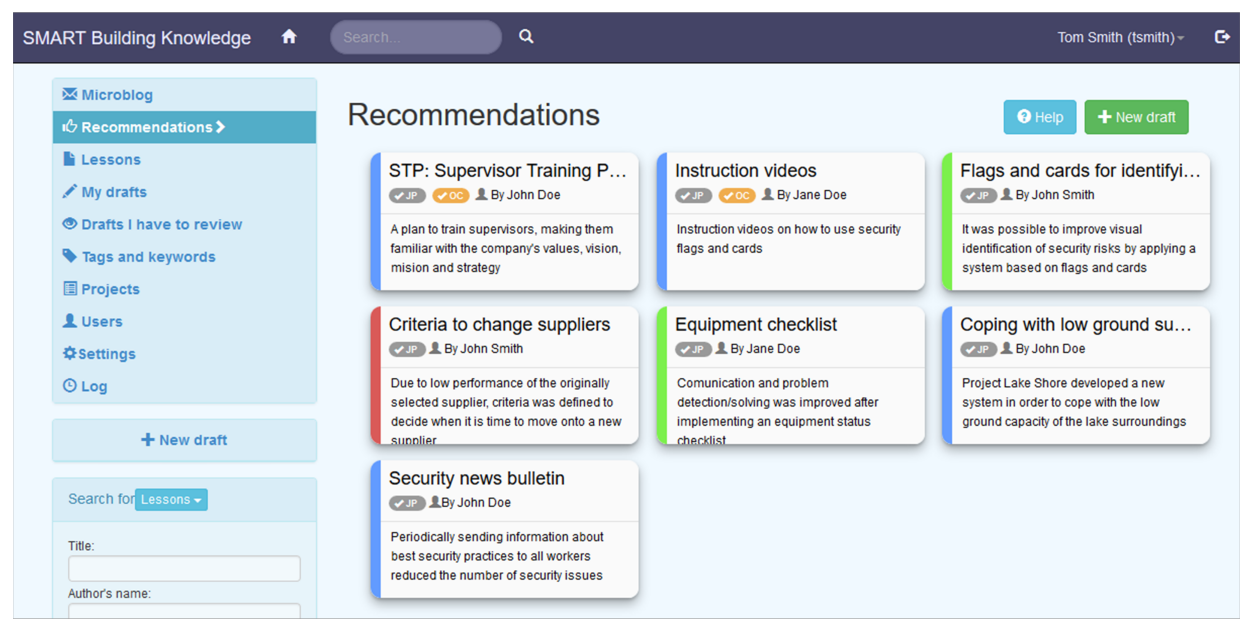

Figure 7. Front-end web application for the content-based recommendation system.

\subsection{Mobile Application}

The mobile application aims to achieve a quick and easy field data collection. In addition, timely access to LL already published in the platform, thereby supporting decision making by focusing primarily on requirements R1 and R2. To do this, it presents a simplified view of the core functionalities explained above, taking advantage of the mobile devices own capabilities, also taking into account R11. For example, in order to explain a certain situation, it is not necessary to write anything, even though this alternative exists. Instead, it is possible to easily take and upload a photo or an audio note from the application (R8) and associate this resource to a particular LL draft that can later be processed using the Web application.

\subsubsection{Cross-Platform Implementation}

In order to provide easy access to information to all the relevant participants in the KM process, the application cannot be restricted to a certain mobile OS. The application should be able to be used from at least the more popular devices, consistent with both R11 and R12. The mobile application was, for this reason, implemented using a multiplatform paradigm. Some of the advantages of this paradigm are that it can overcome the constraint of utilizing different languages and frameworks for each platform. Moreover, it takes advantage of knowledge and expertise already gained by programmers and it makes it possible to have "develop once, deploy anywhere" development cycles and applications that can be distributed through a variety of marketplaces [85].

The platform chosen to implement the cross-platform application was Xamarin Forms, as it is able to overcome one of the main concerns of multiplatform development: the fact that its access to some features of the mobile device may be limited. Xamarin claims its applications have access to the full spectrum of functionality exposed by the underlying platform and device, including platform-specific capabilities like iBeacons and Android Fragments, and their performance is comparable to native applications [86]. With the results of this research, we have developed applications for Android, iPhone, and Windows Phone using Xamarin Forms. These three mobile applications use native user controls, a native user interface, native camera access (photo and video), and native access to Global Positioning System (GPS) and maps. This has been accomplished by writing very little platform-specific code $(85 \%$ of the code was reused and 15\% was platform-specific), which allowed the rapid development of the three applications and offered a native, yet similar, experience on the three platforms, as shown in Figure 8. 

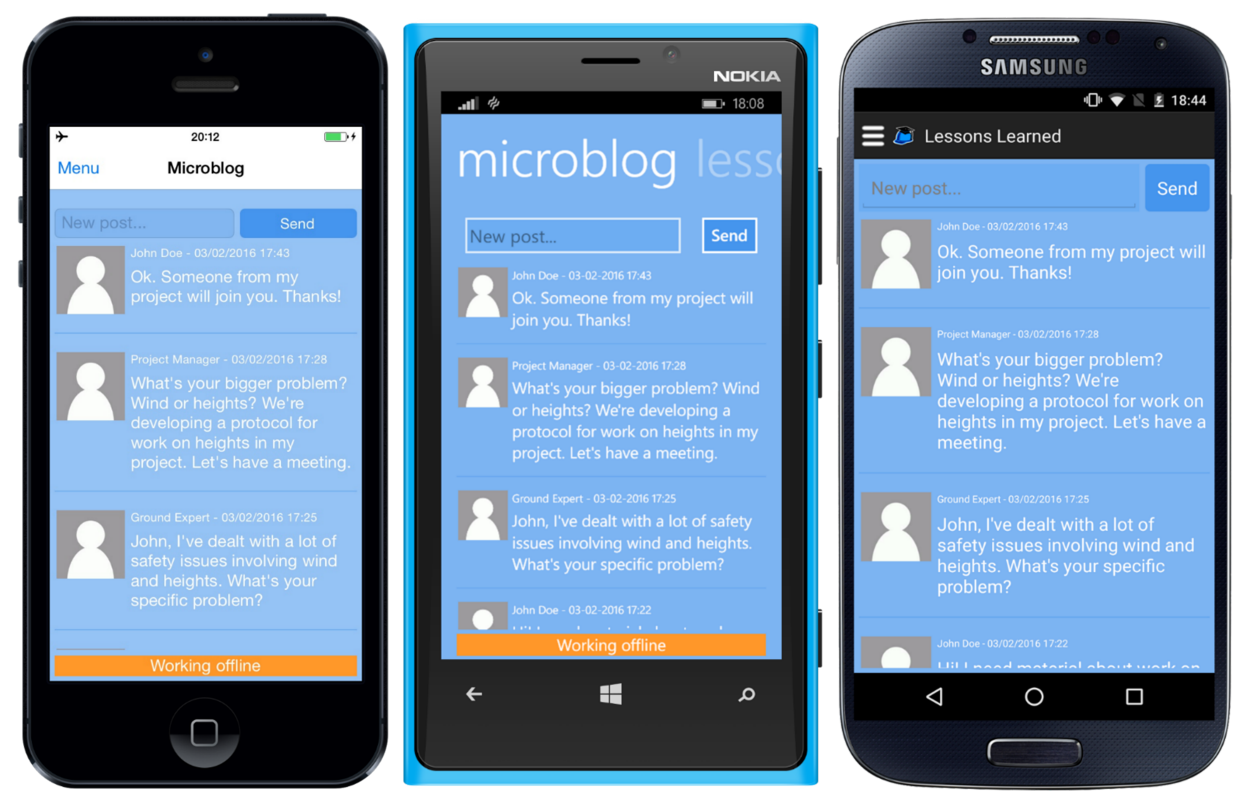

Figure 8. Native, yet similar, Microblog UIs for iOS, Windows Phone, and Android.

\subsubsection{Offline Usage and Data Synchronization}

The mobile application is meant to be used by on-site workers (R1 and R2). The work context may change frequently due to the user's movement or changes in the features of the physical location where the user is. Hence, the mobile application must detect changes in the work context and adapt its services based on them [87]. In this regard, the most important context change for mobile construction professionals is the availability of an Internet connection, as Wi-Fi signals are usually highly unstable on construction sites and mobile Internet is not guaranteed (the worker may or may not have a data plan or broadband providers may lack coverage in specific areas).

Therefore, the mobile application has to be able to function properly with an unstable Internet connection without sacrificing the consistency and availability of the information shared, following R4. This issue is addressed using the software design pattern called "strategy". Figure 9 shows the sequence diagram for the synchronization process between the mobile application and the cloud service. When the device is connected to the Internet, it uses the cloud repository, which sends information directly to the server. When it is not connected, it uses the local repository, which stores the changes locally. When the connection is restored, the local repository tries to synchronize with the cloud repository. To ensure the consistency of the information, if any irreconcilable discrepancy is detected, the user has the final decision on what version of the information should be preserved. 


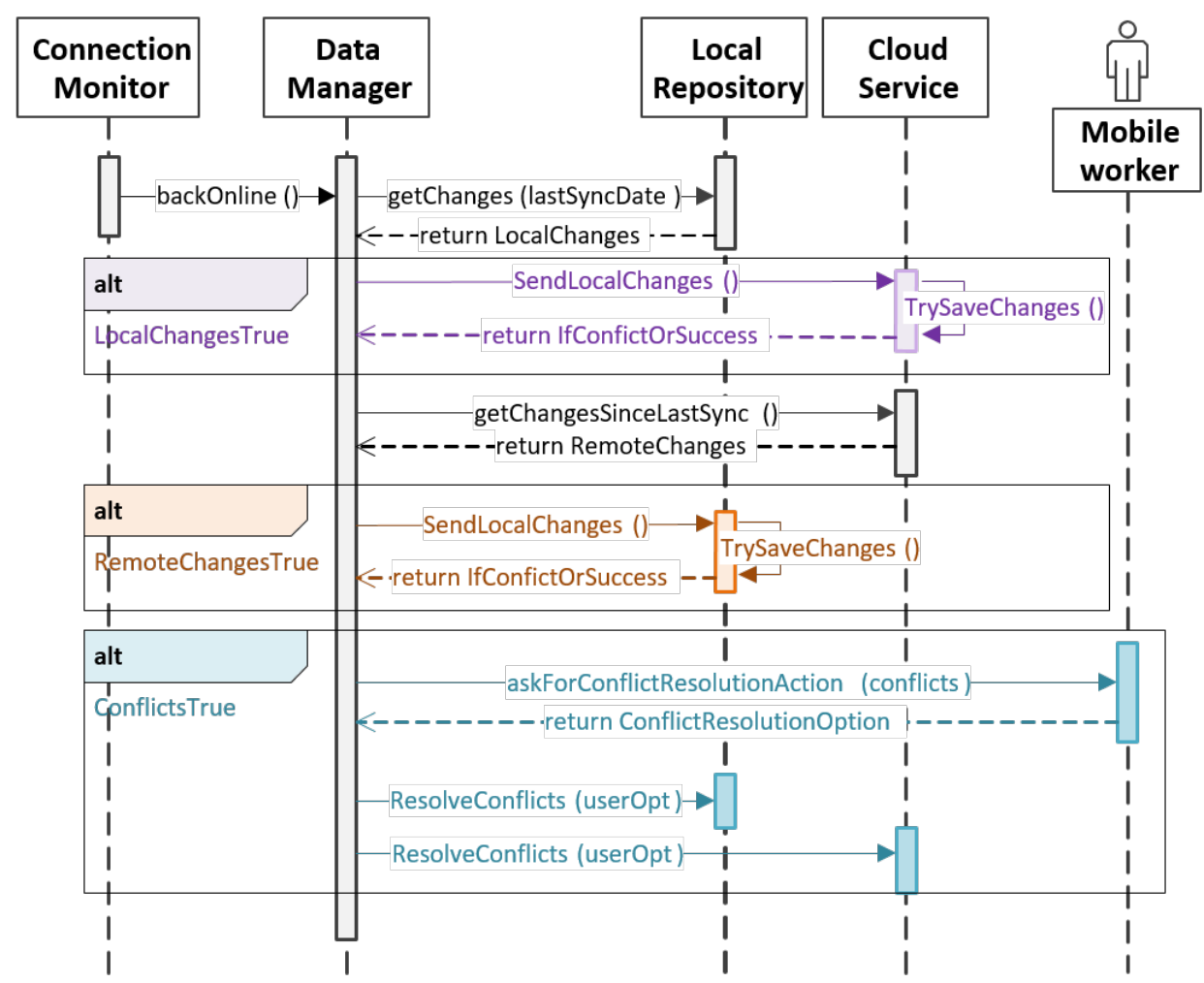

Figure 9. Sequence diagram for the mobile synchronization process.

\subsubsection{Cloud Backend as a Service}

Through the interviews conducted in the Build-Measure-Learn cycles, it became clear that Chilean SME construction companies tend to buy and maintain their own servers as well as all the infrastructure needed to do so. With this strategy, they aim to work without the risk of losing or leaking sensitive data, but other problems emerge because ICT is not their core know-how. Thus, CC becomes an attractive and flexible option to support the application backend as it is secure and makes it possible to use powerful ICT Infrastructure as a Service.

The implementation of this BaaS version is based on different cloud services (web and worker roles). In Azure, a cloud service role is a collection of managed, load-balanced, Platform-as-a-Service virtual machines that work together to perform common tasks. Cloud Service roles are managed by Azure fabric controller and provide the ultimate combination of scalability, control, and customization. Thus, this implemented solution coordinates existing technologies in order to achieve consistent storage and sharing of the knowledge in a way that is simple for both the ICT department and construction professionals, relying on external cloud services provided by powerful vendors, but still giving a high level of control to the construction company than a traditional SaaS. Figure 3 shows the architecture proposed where the core features of the KM platform are built into a piece of software that is deployed in cloud services, forming a cloud backend that can be accessed through Application Programming Interfaces (APIs) by different applications (e.g., mobile and web applications). For instance, the mobile API aims to send and receive the least amount of kilobytes of information possible, to counteract the effect of an Internet connection which will probably be slow or intermittent. Both mobile and web APIs communicate through a cloud-based centralized business logic layer, which is the coordination component and the only one with direct access to the database, information sources, and data processing resources. This architecture can be easily extended to support future new devices with an Internet connection, either by writing applications that consume the existing APIs or by adapting new services. With this, consistency of the information is achieved as all business logic and data is centralized. 
In this schema, the construction company sees the KM platform as a SaaS most of the time, which means that they do not have to constantly worry about maintaining the ICT infrastructure (i.e., updating virtual machines' OS or applying security patches), because such tasks are performed by the cloud vendor. Nevertheless, unlike other software offered using the SaaS model, the construction company ICT department has the ability to alter the features of the cloud platform being used (i.e., boost computing power on certain periods, replicate instances of the KM application, and switch between data centers) because the construction company owns the cloud environment through a cloud subscription. In other words, the construction company uses the KM platform as a black-box, but they do not lose control over what is inside this black-box. Thus, this architecture also makes it possible to consider easily expanding the system in the future, as CC has superior and flexible storage and processing capabilities that are accessible on-demand and not by the physical installation of new servers.

These CC capabilities can be scaled enough to make possible the inclusion of storage and process intensive functionalities such as machine learning [88]. Based on the results of the third research cycle, we decided to incorporate machine learning and content-based recommendations into our solution. Currently, we have the first release of this feature implemented with Azure Machine Learning. This feature in Azure enables machine learning solutions to easily build, deploy, and share predictive analytics, being very useful for this particular KM platform as a tool to improve the knowledge and information management process.

From the construction industry's perspective, this automation reduces the time that professionals have to spend searching for relevant information. Content-based Recommender Systems provide recommendations to users based on comparing items or products to the items that a user showed interest in. A user profile in these systems represents explanations of product characteristics that the user chose before. These explanations are illustrated by a set of characteristics or features describing the products in a user profile. The act of producing recommendations usually includes comparing features of items unseen or unrated by the user with his or her profile content description. The items that are similar enough to the user's profile are recommended to him or her [83]. Many Recommender Systems are centered around the use of various machine learning and data mining algorithms to predict user evaluations for items, or for learning how to correctly rank items for a user [89].

Figure 10 shows the current version of the cloud-based lesson recommendation system that aims to group the LL available in the system and to map the user's behavior and preferences to such clusters. To achieve such a task, the LL is represented by vectors in a $\mathrm{N}$-dimensional space, where $\mathrm{N}$ is the number of features to be considered for their classification. The format of the LL proposed by Ferrada et al. [57] and used in this cloud-based mobile platform is a mix of structured and unstructured data, which is important to consider in the formation of these vectors. The fields for lessons triggers and disciplines are examples of structured data, as they have to be selected from a list predetermined by the system administrator. These features can be directly represented in a vector. On the other hand, the fields for case description and learning are examples of unstructured data, as they are unrestricted texts that can exhibit the full complexity of natural language [90]. A common approach to dealing with free text fields is to convert the free text to a structured representation. For example, each word may be viewed as an attribute, with a Boolean value indicating whether the word is in the article or with an integer value indicating the number of times the word appears in the text [90]. Nevertheless, at the time of consolidating the attribute space of all lessons, such an approach induces a sparse matrix because words that may be present in one lesson will not necessarily be present in others and the word length of the texts is variable. To reduce this dimensionality, Principal Component Analysis (PCA) can be used [91], resulting in a representation of the free texts that has substantially fewer dimensions and is more sparse than using the whole spectrum of different words present in them. The N-dimensional space will be the sum of the dimensions from the structured data and the unstructured data after PCA. 


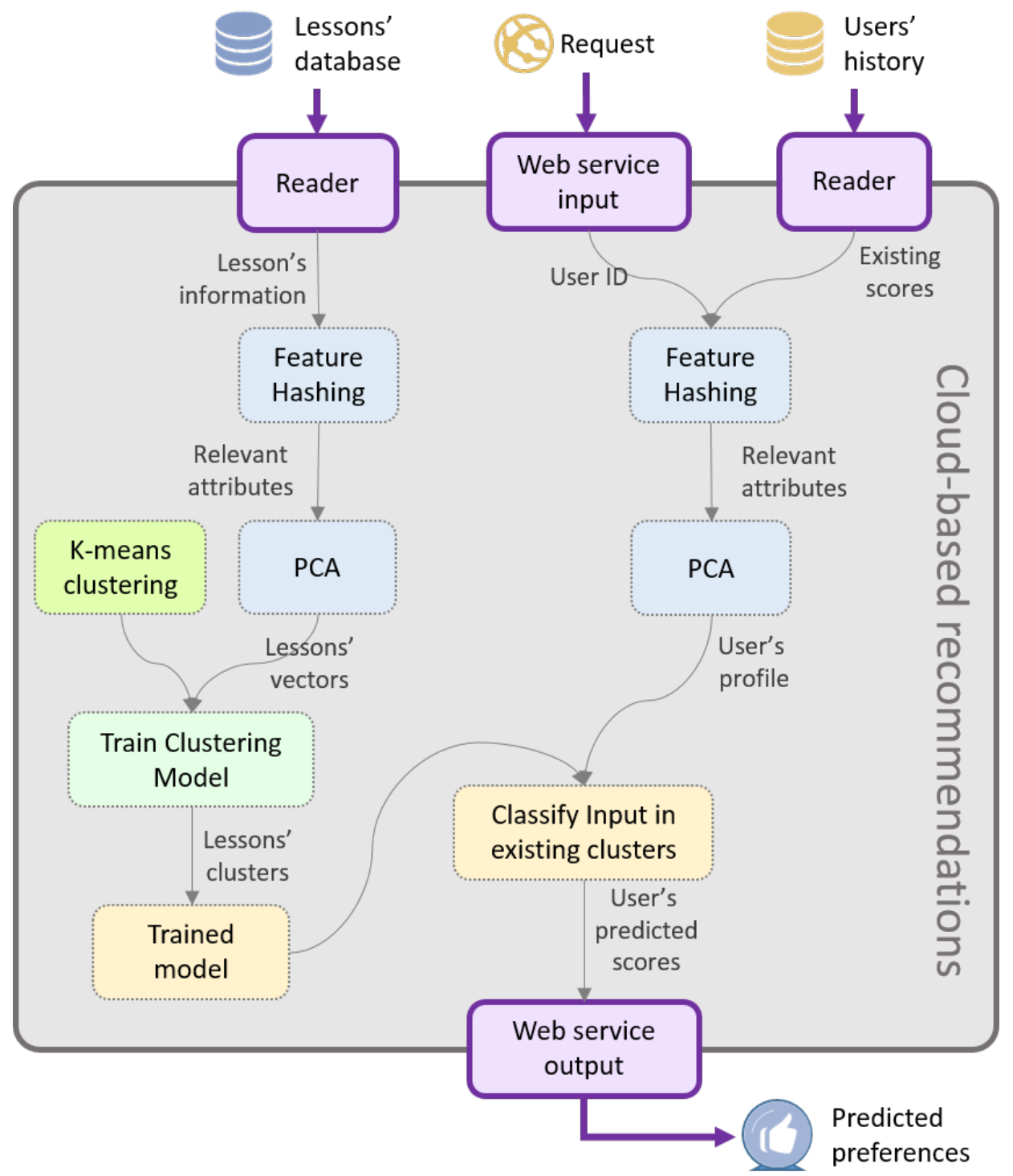

Figure 10. Cloud-based lesson recommendations.

Finally, when the lessons are represented as vectors, they are grouped by similarity using K-mean clustering, which uses an iterative algorithm that minimizes the sum of distances from each object to its cluster centroid, over all clusters. The algorithm moves objects between clusters until the sum cannot be decreased any further [91]. To map the user's preferences to the resulting clusters, the profile of each user is built considering the lessons he or she had written or approved and his or her most read lessons and/or topics. This information allows the scoring of each cluster of lessons. Then, the system recommends unread lessons from all clusters, prioritizing lessons from clusters with the highest scores for that user.

\section{Platform Evaluation}

The second and third Build-Measure-Learn cycles produced functional prototypes of the platform. Therefore, the three-part test described in Section 4.3. was applied twice, once for each functional version of the platform. Sections 6.1 and 6.2 present the results of these tests, respectively. 


\subsection{Second Cycle Test-Platform Core Features}

During the second Build-Measure-Learn cycle, this research used an early prototype of the cloud-based mobile platform to conduct an evaluation of the platforms reception and potential impact within one of the construction companies that participated in the first research cycle. This pilot study allowed professionals from the same project team to interact with the system according to their own roles and to evaluate all of its functionalities using a simple Web application and a prototype of the mobile application.

The selected project team included a supervising manager, a project manager, a site manager, a technical office professional, a technical office assistant, and the Management and Innovation Head of the company. This team was working on the project of finishing the common spaces $\left(2400 \mathrm{~m}^{2}\right)$ of a major office building. Because of the finely detailed nature of the work to be done on this project, it was considered an excellent source of LL.

Table 4 shows the scores resulting from the first and second parts of the questionnaire. Regarding the open questions, all respondents indicated that the platform could improve their company's KM process on-site. They based their statements on the fact that the platform is user-friendly and that it allows for a knowledge repository to learn from successful ideas and previous problem resolutions that avoid duplicating a solution that has already previously been created or optimized by others. As pointed out by the supervising manager when asked if he would use the KM platform: "Yes, because it is friendly and simple. I think it can be acquired as a work tool by the professionals of the company". In the same way, the site manager said: "Of course, since it allows providing feedback about solutions associated to operation deviations in a simple, practical and orderly manner, interacting with different levels of the organization", while the technical office professional indicated: "Yes, it is good to have a place where you can find problems or learnings so as not to make the same mistakes, and be able to copy good ideas".

Table 4. First prototype scores from the first and second parts of the test.

\begin{tabular}{cccc}
\hline Evaluator & SUS Score & $\begin{array}{c}\text { ISO-Quality Systems and } \\
\text { Software Total Score (TS) }\end{array}$ & $\begin{array}{c}\text { ISO-Quality Systems } \\
\text { and Software TS/QA }\end{array}$ \\
\hline A & 92.5 & 49 & 4.5 \\
B & 90.0 & 52 & 4.7 \\
C & 85.0 & 48 & 4.4 \\
D & 65.0 & 42 & 3.8 \\
E & 62.5 & 38 & 3.5 \\
F & 87.5 & 51 & 4.6 \\
\hline Average & 80.42 & 46.67 & 4.25 \\
\hline
\end{tabular}

As regards the implementation of the platform in the company, interviewees indicated some concerns, such as the IT capabilities of people and systems in the company and the Internet connection speed on-site. Given the field conditions, it could be unstable, affecting the platform performance. Regarding the limitations for its adoption, the project manager pointed out: "Internet access can be an impediment; if it is deficient it becomes less motivating". The technical office professional said: "The main problem may be the age of the users and the low level of computational skill they have, compared to the use of the younger ones", while the supervising manager indicated: "In the field, the mobile phones that are assigned to the professionals do not work for this application, you have to make a change on that. Also you have to check the speed of Internet and browser versions on-site. A good filter is important so as it does not fill with useless information".

Finally, to improve the usability of the platform, interviewees pointed out that it could be good to, include e-mail notifications for different platform tasks; improve the graphical environment of the webpage, including a profile picture for the project and more colors and graphics; add an auto save every minute; improve labels and search facilities; and to try to foster a business culture and openness 
to innovation in the company. The above is based on the comments of construction professionals, where, for example, the supervising manager stated: "E-mail notifications are required for approvals or other communications. The design of the page could be more innovative, youthful, cheerful, more colorful, with more graphical design". The project manager, meanwhile, indicated: "improve the graphic environment of the page and auto-save programmed every minute".

\subsection{Third Cycle-Mobile Application With Cloud Backend}

During the third Build-Measure-Learn cycle, this research developed a more elaborate version of the cloud-based mobile platform and conducted an evaluation of it, consulting an expert panel. This panel was composed of seven professionals in the architecture, engineering and construction sectors and included experts in construction management, sustainability, and architecture.

This evaluation allowed the panel to interact mainly with the mobile application, simulating a situation in which a project team could use both the microblogging and LL platforms on-site. They tested both the online and the offline mode. Finally, they tested the consistency of the information entered through the mobile application with what was shown to them in the Web application.

Table 5 shows the scores resulting from the first and second parts of the questionnaire. Regarding the open questions, all respondents indicated that the platform has the potential to improve the LL process in construction companies because it provides systematization of a process that has not yet been formalized in SMEs. They also identified some threats to the correct implementation and adoption of this cloud-based mobile platform. They were mainly concerned about the resistance to change present in the construction companies' organizational culture. Despite this concern, they were optimistic and suggested possible ways to address such issues, for instance, to financially quantify the benefits of having each lesson (or the extra expenses of not having it earlier), to provide incentives to those who use the platform regularly, and/or to allow this platform to communicate with other systems that have already been implemented within the company (e.g., Office 360 or BIMx).

They also saw great potential in the proposed data-processing features because, in their opinion, they turned the platform into an active and useful agent within the project team rather than a burden imposed by headquarters. They even suggested improvements to the machine learning features. For instance, one of the experts who specializes in management suggested automatically generating "quick start bundles" of lessons for projects that are just starting, using project profiles as well as user profiles in the recommendation system.

Table 5. Expert panel scores from the first and second parts of the test.

\begin{tabular}{cccc}
\hline Evaluator & SUS Score & $\begin{array}{c}\text { ISO-Quality Systems and } \\
\text { Software Total Score (TS) }\end{array}$ & $\begin{array}{c}\text { ISO-Quality Systems and } \\
\text { Software TS/QA }\end{array}$ \\
\hline G & 92.5 & 51 & 4.64 \\
H & 87.5 & 54 & 4.91 \\
I & 82.5 & 49 & 4.45 \\
J & 87.5 & 51 & 4.64 \\
K & 90.0 & 48 & 4.36 \\
L & 70.0 & 52 & 4.73 \\
M & 75.0 & 51 & 4.64 \\
\hline Average & 83.57 & 50.86 & 4.62 \\
\hline
\end{tabular}

\subsection{Limitations}

Although the KM platform was well evaluated by users, it still has some limitations. First, the platform was designed according to the information gathered in three construction SMEs that belong to the medium-sized category and none of them to the small-sized category, which may have left outside the platform some relevant requirements for this type of construction companies. Second, at present the platform does not integrate with other information systems that a company could be 
using to manage a project, such as planning software or BIM systems, which may discourage its use. Finally, the validation of the platform in the field involved a team of professionals working on the completion of an important office building in a downtown area of the city, where connectivity and Internet access would be expected to be better than in other sectors away from the downtown area or on the outskirts of the city, which could affect the results of the evaluation of the KM platform.

To face these limitations and improve the KM platform, it would be necessary to carry out a new validation process incorporating a group of small-sized construction companies, to verify that the system responds adequately to their needs, or to incorporate the necessary improvements. On the other hand, the KM platform should be tested in projects farther away from central or populated areas, to evaluate its performance under more extreme connectivity conditions, for both small and medium-sized companies. Finally, regarding the integration with other information systems, it would be necessary to evaluate, together with the professionals in the area, which systems are most used and what integration needs exist in order to incorporate them into the KM platform.

Although MCC is a promising alternative for the needs of SMEs, there are some challenges that still remain to be solved, among them are: limited hardware resources and energy consumption in mobile devices, computational offloading, connectivity, security, latency, and heterogeneity $[92,93]$. These challenges are discussed by these authors, including how they are currently being addressed. Some of these limitations were considered in the design of the proposed KM platform, such as limited computational capacity, battery power consumption, connectivity and heterogeneity. Others remain as open issues, such as security and latency.

Even though the research was carried out in Chilean construction SMEs, the results of the interviews carried out to understand the way in which the knowledge generated in the projects is managed are not different from those described in the literature for this type of companies. In general, they have poorly structured systems, where much depends on tacit knowledge, and the use of KMSs is infrequent. Therefore, it is not considered that the national context may have affected the results. As described in the literature review, the problems of construction SMEs are similar in other countries, so the results of this research have the potential to be replicable in other countries. Moreover, the technologies used (MCC) facilitate its adoption, since they do not require a local investment in technology (at the server level) and use mobile technology in the construction sites, which has an exponential adoption worldwide.

\section{Conclusions and Further Work}

This article presents a novel MCC-based mobile platform to support the knowledge management process of SMEs in the Chilean construction industry. The approach applied allowed us to encapsulate the complex design decisions and trade-offs involved in the architecture of a mobile application with augmented capabilities via cloud backend services destined for improving knowledge management in SMEs construction companies. The KM platform was built with different cloud services provided by Microsoft Azure. The platform has been considered by users as a useful tool with great potential to improve the performance of the knowledge management process, as it provides systematization of a process that has not yet been formalized in Chilean SMEs.

To develop the platform, different research cycles were performed divided into three distinct phases (Build-Measure and Learn) according to the lean development methodology. This research methodology allows for the development of a platform that focuses on the specific needs and requirements of SMEs in Chilean construction companies, recognizing the particularities of their work and their cultural characteristics, as they are relevant factors to take into account in order to have a successful system. In this way, this research addresses a gap as similar systems may be MCC-based, may focus on KM process, may be designed for construction companies and/or SME's, but do not simultaneously have these four qualities. According to the positive result of the evaluations, this research concludes that the proposed platform is already responding to the needs of this sector using the trending paradigm of MCC, thus helping to close the research gap identified at the beginning 
of this article. This was achieved by constantly testing and improving the hypothesis of the system and the research through research cycles.

Regarding the implementation of this MCC-based platform, this study is able to conclude that using a cloud backend combined with mobile applications is a way of enhancing SMEs KM by providing this process with powerful data storage and data processing capabilities at an acceptable level of cost and risk. Shifting large volumes of data to the cloud provides a flexible and scalable platform for developing a variety of scientific data-processing tasks. MCC is then, a very attractive option for KM in construction SMEs from multiple perspectives. For instance, the mobility introduced by the mobile application helps with the reutilization of past experiences on-site. Moreover, it is financially attractive because these services may be hired at prices that fit the needs and size of the company. Besides this, CC capabilities supporting the backend functionality are expected to be better than an in-house solution, as they are provided by specialized vendors instead of being deployed by IT departments that, most likely, are not experts in the subject.

In terms of the mobile application itself, the use of a cross-platform framework in the construction industry domain is feasible. A cross-platform framework meets the requirements of mobile applications with non-complex UIs and accelerates the development of applications targeting the most popular mobile markets. Cross-platform development also makes it possible to offer a similar user experience for most mobile users and helps to maintain the consistency of the information and business logic, as most of the code is reused on all platforms. In addition, if the framework allows the code to be deployed in native applications (as Xamarin.Forms does), performance is not hindered. These conclusions are supported by the test results of the third cycle.

In terms of offline usage, systems targeting the construction sector must have an offline mode that allows access to the most recent copies of any important information available and synchronization with the centralized servers (e.g., cloud backend) once connection is restored because maintaining a stable Internet connection is almost impossible on construction sites.

Lastly, it is important to note that, as pointed out by construction experts, the most relevant threat to the success of the platform is faulty change management rather than some failure in the platform itself. Therefore, when this platform reaches industry, it has to be accompanied by proper training and incentives to overcome this initial barrier. Nevertheless, this barrier could be minimized by addressing this challenge from a multidisciplinary perspective and by taking into account the feedback from user evaluations (e.g., ease of use, validation process, and user's expectations). The positive results already obtained in the evaluations by end users give this platform a head-start in this matter.

Even though the platform received positive comments from users, the last evaluation showed that minor corrections need to be done to the platform before it can be rolled out to industry. Also, it showed that cloud computing capabilities can be further exploited. In order to do so, we have a first release of a recommender system relying on machine learning developed in a fourth Build-Measure-Learn cycle. It is expected that this automation will reduce the time that professionals have to spend searching for relevant information as it will provide recommendations to users based on comparing new lessons to the lessons the user previously showed an interest in. In the future, we expect to add news features (like a chatbot able to recommend lessons through translating and interpreting human natural language input) to this new recommender system of the platform, so as to evaluate its usefulness on site.

Additionally, we want to consider a more functional integration with BIM objects. Currently, the platform allows for interaction with BIM models through the files that can be attached to a lesson, which is a very simple form of integration because few Chilean construction SMEs have fully adopted BIM so far, but it is expected that this situation change in the future. Any future improvements should take advantage of the extensibility of the proposed platform to add a richer integration with BIM.

In addition, open issues and challenges in the technologies that were used to build the platform (i.e., MC and CC), such as data security, need to be studied in further detail. This will ensure that this proposal is kept up-to-date with state of the art research as advances are made. 
Finally, we believe that this proposal will contribute to the generation of the conceptual basis and foundations of a knowledge management platform architecture to manage lessons learned. A platform especially designed for Chilean SME construction companies that use the cloud in an effective and efficient way. Besides the obvious academic contribution of the proposed research, we expect that this platform could produce concrete changes. First in the construction industry (better and more efficient solutions) as it will help companies to improve their knowledge management processes thereby allowing for a better use of the lessons learned on each project, reducing the repetition of mistakes, and enabling the creation of a learning culture inside each organization. Second in the productivity of construction professionals, as it will help them to have access at any time and from anywhere to organizational knowledge in order to enhance their performance at work.

Acknowledgments: The authors wish to acknowledge CONICYT FONDECYT/Postdoctorado/N: 3130412 for the support for the realization of this research effort.

Author Contributions: Ximena Ferrada developed the research presented in this study as part of a postdoctoral project supervised by Marcos Sepúlveda and Alfredo Serpell. Andrés Neyem contributed in the design of the proposed system and in the supervision of Daniela Núnez, who developed the computational platform presented in this article. All authors wrote this article.

Conflicts of Interest: The authors declare no conflict of interest.

\section{References}

1. Egbu, C.O.; Robinson, H.S. Construction as a knowledge-based industry. Knowl. Manag. Constr. 2005, 4, 31-49.

2. Kim, S. Impacts of knowledge management on the organizational success. KSCE J. Civ. Eng. 2014, 18, 1609-1617.

3. North, K.; Babakhanlou, R. Knowledge Management Tools for SMES. In Competitive Strategies for Small and Medium Enterprises; Springer: Cham, Switzerland, 2016; pp. 211-222.

4. Production Development Corporation (CORFO). Hoja de Ruta Programa Estratégico Nacional Productividad y Construcción Sustentable 2025, Informe Final, Fase 3; Technical Report; CORFO: Santiago, Chile, 2016.

5. Wong, K.Y.; Aspinwall, E. Development of a knowledge management initiative and system: A case study. Expert Syst. Appl. 2006, 30, 633-641.

6. Alavi, M.; Leidner, D.E. Knowledge management and knowledge management systems: Conceptual foundations and research issues. MIS Q. 2001, 25, 107-136.

7. Cerchione, R.; Esposito, E. A systematic review of supply chain knowledge management research: State of the art and research opportunities. Int. J. Prod. Econ. 2016, 182, 276-292.

8. Alvarez, I.; Cilleruelo, E.; Zamanillo, I. Is formality in knowledge management practices related to the size of organizations? The Basque case. Hum. Factors Ergon. Manuf. Serv. Ind. 2016, 26, 127-144.

9. Duffield, S.; Whitty, S.J. Developing a systemic lessons learned knowledge model for organisational learning through projects. Int. J. Proj. Manag. 2015, 33, 311-324.

10. Ling, C.T.N. Knowledge management acceptance: Success factors amongst small and medium-size enterprises. Am. J. Econ. Bus. Adm. 2011, 3, 73-80.

11. Cheng, J.C.; Kumar, B. Cloud computing support for construction collaboration. Mob. Pervasive Comput. Constr. 2012, 237-254, doi:10.1002/9781118422281.ch12.

12. Ahuja, V.; Yang, J.; Skitmore, M.; Shankar, R. An empirical test of causal relationships of factors affecting ICT adoption for building project management: An Indian SME case study. Constr. Innov. 2010, 10, 164-180.

13. Lin, K.Y.; Tsai, M.H.; Gatti, U.C.; Lin, J.J.C.; Lee, C.H.; Kang, S.C. A user-centered information and communication technology (ICT) tool to improve safety inspections. Autom. Constr. 2014, 48, 53-63.

14. Poppendieck, M.; Poppendieck, T. Implementing Lean Software Development: From Concept to Cash; Pearson Education; Addison-Wesley: Boston, MA, USA, 2007.

15. Ho, S.P.; Tserng, H.P.; Jan, S.H. Enhancing knowledge sharing management using BIM technology in construction. Sci. World J. 2013, 2013, 170498.

16. Deshpande, A.; Azhar, S.; Amireddy, S. A framework for a BIM-based knowledge management system. Procedia Eng. 2014, 85, 113-122. 
17. Nepal, M.P.; Staub-French, S. Supporting knowledge-intensive construction management tasks in BIM. J. Inf. Technol. Constr. 2016, 21, 13-38.

18. Muñoz, V.; Arayici, Y. Using free tools to support the BIM coordination process into SMEs. Build. Inf. Model. Design Constr. Oper. 2015, 149, 33-41.

19. Loyola, M. Encuesta Nacional BIM 2016: Informe de Resultados; Technical Report; Universidad de Chile: Santiago, Chile, 2016.

20. Avram, M.G. Advantages and challenges of adopting cloud computing from an enterprise perspective. Procedia Technol. 2014, 12, 529-534.

21. Stieglitz, S.; Lattemann, C.; Brockmann, T. Mobile applications for knowledge workers and field workers. Mob. Inf. Syst. 2015, 2015.

22. Wang, Y.; Chen, R.; Wang, D.C. A survey of mobile cloud computing applications: Perspectives and challenges. Wirel. Pers. Commun. 2015, 80, 1607-1623.

23. Abolfazli, S.; Sanaei, Z.; Sanaei, M.H.; Shojafar, M.; Gani, A. Mobile Cloud Computing. In Encyclopedia of Cloud Computing; Wiley-IEEE Press: Hoboken, NJ, USA, 2016; pp. 29-40.

24. Tarchinskaya, E.; Taratukhin, V.; Becker, J. Cloud-Based Engineering Design and Manufacturing: A Survey. In Emerging Trends in Information Systems; Springer: Cham, Switzerland, 2016; pp. 125-135.

25. Du, J.; El-Gafy, M.; Lama, P. A Cloud-based shareable library of cooperative behaviors for Agent Based Modeling in construction. Autom. Constr. 2016, 62, 89-100.

26. Ibem, E.O.; Laryea, S. Survey of digital technologies in procurement of construction projects. Autom. Constr. 2014, 46, 11-21.

27. Chong, H.Y.; Wong, J.S.; Wang, X. An explanatory case study on cloud computing applications in the built environment. Autom. Constr. 2014, 44, 152-162.

28. Kim, C.; Park, T.; Lim, H.; Kim, H. On-site construction management using mobile computing technology. Autom. Constr. 2013, 35, 415-423.

29. Ko, H.S.; Azambuja, M.; Lee, H.F. Cloud-based materials tracking system prototype integrated with radio frequency identification tagging technology. Autom. Constr. 2016, 63, 144-154.

30. Moon, S.; Xu, S.; Hou, L.; Wu, C.; Wang, X.; Tam, V.W. RFID-Aided Tracking System to Improve Work Efficiency of Scaffold Supplier: Stock Management in Australasian Supply Chain. J. Constr. Eng. Manag. 2017, 144, 04017115.

31. Autodesk Inc. Autodesk BIM 360. Available online: http://bim360.autodesk.com (accessed on 29 January 2018).

32. Graphisoft. BIMx. Available online: http://www.graphisoft.com/bimx (accessed on 29 January 2018).

33. PlanGrid. PlanGrid. Available online: http://www.plangrid.com (accessed on 29 January 2018).

34. Autodesk Inc. A360. Available online: https:/ / a360.autodesk.com (accessed on 29 January 2018).

35. Slack. Slack. Available online: https://slack.com (accessed on 29 January 2018).

36. XWiki. XWiki. Available online: http://www.xwiki.org/xwiki/bin/view/Main/WebHome (accessed on 29 January 2018).

37. AnswerHub. Available online: http://www.dzonesoftware.com (accessed on 29 January 2018).

38. Centobelli, P.; Cerchione, R.; Esposito, E. Knowledge management in startups: Systematic literature review and future research agenda. Sustainability 2017, 9, 361.

39. Durst, S.; Runar Edvardsson, I. Knowledge management in SMEs: A literature review. J. Knowl. Manag. 2012, 16, 879-903.

40. Cerchione, R.; Esposito, E.; Spadaro, M.R. The spread of knowledge management in SMEs: A scenario in evolution. Sustainability 2015, 7, 10210-10232.

41. Cerchione, R.; Esposito, E.; Spadaro, M.R. A literature review on knowledge management in SMEs. Knowl. Manag. Res. Pract. 2016, 14, 169-177.

42. Cerchione, R.; Esposito, E. Using knowledge management systems: A taxonomy of SME strategies. Int. J. Inf. Manag. 2017, 37, 1551-1562.

43. Wong, K.Y.; Aspinwall, E. An empirical study of the important factors for knowledge-management adoption in the SME sector. J. Knowl. Manag. 2005, 9, 64-82.

44. Giotopoulos, I.; Kontolaimou, A.; Korra, E.; Tsakanikas, A. What drives ICT adoption by SMEs? Evidence from a large-scale survey in Greece. J. Bus. Res. 2017, 81, 60-69. 
45. Centobelli, P.; Cerchione, R.; Esposito, E. Knowledge management systems: The hallmark of SMEs. Knowl. Manag. Res. Pract. 2017, 15, 294-304.

46. Centobelli, P.; Cerchione, R.; Esposito, E. Aligning enterprise knowledge and knowledge management systems to improve efficiency and effectiveness performance: A three-dimensional Fuzzy-based decision support system. Expert Syst. Appl. 2018, 91, 107-126.

47. Priyadarshinee, P.; Raut, R.D.; Jha, M.K.; Kamble, S.S. A cloud computing adoption in Indian SMEs: Scale development and validation approach. J. High Technol. Manag. Res. 2017, 28, 221-245.

48. Love, P.E.; Ackermann, F.; Teo, P.; Morrison, J. From individual to collective learning: A conceptual learning framework for enacting rework prevention. J. Constr. Eng. Manag. 2015, 141, 05015009.

49. Castro, A.; Yepes, V.; Pellicer, E.; Cuellar, A. Knowledge management in the construction industry: State of the art and trends in research. Revista de la Construcción 2012, 11, 62-73.

50. Yang, L.R.; Chen, J.H.; Wang, H.W. Assessing impacts of information technology on project success through knowledge management practice. Autom. Constr. 2012, 22, 182-191.

51. Tan, H.C.; Carrillo, P.M.; Anumba, C.J.; Bouchlaghem, N.; Kamara, J.M.; Udeaja, C.E. Development of a methodology for live capture and reuse of project knowledge in construction. J. Manag. Eng. 2007, 23, 18-26.

52. Dave, B.; Koskela, L. Collaborative knowledge management-A construction case study. Autom. Constr. 2009, 18, 894-902.

53. Bowden, S.; Dorr, A.; Thorpe, T.; Anumba, C. Mobile ICT support for construction process improvement. Autom. Constr. 2006, 15, 664-676.

54. Tan, H.C.; Carrillo, P.M.; Anumba, C.J. Case study of knowledge management implementation in a medium-sized construction sector firm. J. Manag. Eng. 2011, 28, 338-347.

55. Caldas, C.H.; Gibson, G.E., Jr.; Weerasooriya, R.; Yohe, A.M. Identification of effective management practices and technologies for lessons learned programs in the construction industry. J. Constr. Eng. Manag. 2009, 135, 531-539.

56. Eken, G.; Bilgin, G.; Dikmen, I.; Birgonul, M.T. A lessons learned database structure for construction companies. Procedia Eng. 2015, 123, 135-144.

57. Ferrada, X.; Sepúlveda, M.; Serpell, A.; Núñez, D.; Neyem, A. A lessons-learned mobile system for construction companies: motivation and design. Procedia Eng. 2014, 85, 157-165.

58. Forcada, N.; Fuertes, A.; Gangolells, M.; Casals, M.; Macarulla, M. Knowledge management perceptions in construction and design companies. Autom. Constr. 2013, 29, 83-91.

59. Davison, R.M.; Ou, C.X.; Martinsons, M.G. Information technology to support informal knowledge sharing. Inf. Syst. J. 2013, 23, 89-109.

60. Sage. Cloud Computing and the Construction Industry; Technical Report; Sage: Newcastle upon Tyne, UK, 2012.

61. Lin, Y.C.; Wang, L.C.; Tserng, H.P. Enhancing knowledge exchange through web map-based knowledge management system in construction: Lessons learned in Taiwan. Autom. Constr. 2006, 15, 693-705.

62. Tserng, H.P.; Lin, Y.C. Developing an activity-based knowledge management system for contractors. Autom. Constr. 2004, 13, 781-802.

63. Paranagamage, P.; Carrillo, P.; Ruikar, K.; Fuller, P. Lessons learned practices in the UK construction sector: Current practice and proposed improvements. Eng. Proj. Organ. J. 2012, 2, 216-230.

64. Lin, Y.C. Construction 3D BIM-based knowledge management system: A case study. J. Civ. Eng. Manag. 2014, 20, 186-200.

65. Kivits, R.A.; Furneaux, C. BIM: Enabling sustainability and asset management through knowledge management. Sci. World J. 2013, 2013, 983721.

66. Chen, Y.; Kamara, J.M. Using mobile computing for construction site information management. Eng. Constr. Archit. Manag. 2008, 15, 7-20.

67. Du, J.; Shi, Y.; Zou, Z.; Zhao, D. CoVR: Cloud-Based Multiuser Virtual Reality Headset System for Project Communication of Remote Users. J. Constr. Eng. Manag. 2017, 144, 04017109.

68. Rosado da Cruz, A.M. Modern Software Engineering Methodologies for Mobile and Cloud Environments; IGI Global: Hershey, PA, USA, 2016.

69. Thomas, B. Cloud Computing for Construction Accounting; Building Profits; CFMA: Princeton, NJ, USA, 2012.

70. Silverio, M.; Renukappa, S.; Suresh, S.; Donastorg, A. Mobile Computing in the Construction Industry: Main Challenges and Solutions. In Leadership, Innovation and Entrepreneurship as Driving Forces of the Global Economy; Springer: Cham, Switzerland, 2017; pp. 85-99. 
71. Salah, D.; Paige, R.F.; Cairns, P. A systematic literature review for agile development processes and user centred design integration. In Proceedings of the 18th International Conference on Evaluation and Assessment in Software Engineering, London, UK, 13-14 May 2014; p. 5.

72. Brhel, M.; Meth, H.; Maedche, A.; Werder, K. Exploring principles of user-centered agile software development: A literature review. Inf. Softw. Technol. 2015, 61, 163-181.

73. Da Silva, T.S.; Martin, A.; Maurer, F.; Silveira, M. User-centered design and agile methods: A systematic review. In Proceedings of the Agile Conference (AGILE), Salt Lake City, UT, USA, 7-13 August 2011; pp. 77-86.

74. Ries, E. The Lean Startup: How Today's Entrepreneurs Use Continuous Innovation to Create Radically Successful Businesses; Crown Books: Prince George's County, MD, USA, 2011.

75. Silva, S.E.; Calado, R.D.; Silva, M.B.; Nascimento, M. Lean Startup applied in Healthcare: A viable methodology for continuous improvement in the development of new products and services. IFAC Proc. Vol. 2013, 46, 295-299.

76. Carrillo, P.; Ruikar, K.; Fuller, P. When will we learn? Improving lessons learned practice in construction. Int. J. Proj. Manag. 2013, 31, 567-578.

77. Gao, S.; Krogstie, J.; Siau, K. Adoption of mobile information services: An empirical study. Mob. Inf. Syst. 2014, 10, 147-171.

78. Brooke, J. SUS-A quick and dirty usability scale. Usabil. Eval. Ind. 1996, 189, 4-7.

79. Brooke, J. SUS: A retrospective. J. Usabil. Stud. 2013, 8, 29-40.

80. Bangor, A.; Kortum, P.T.; Miller, J.T. An empirical evaluation of the system usability scale. Int. J. Hum. Comput. Interact. 2008, 24, 574-594.

81. Tullis, T.S.; Stetson, J.N. A comparison of questionnaires for assessing website usability. In Proceedings of the Usability Professional Association Conference, Minneapolis, MN, USA, 7-11 June 2004; pp. 1-12.

82. ISO. Iec25010: 2011 systems and software engineering-systems and software quality requirements and evaluation (square)-system and software quality models. Int. Organ. Stand. 2011, 34, 2910.

83. Parra, D.; Sahebi, S. Recommender systems: Sources of knowledge and evaluation metrics. In Advanced Techniques in Web Intelligence-2; Springer: Berlin/Heidelberg, Germany, 2013; pp. 149-175.

84. Borchers, A.; Herlocker, J.; Konstan, J.; Reidl, J. Ganging up on information overload. Computer 1998, 31, 106-108.

85. Corral, L.; Janes, A.; Remencius, T. Potential advantages and disadvantages of multiplatform development frameworks-A vision on mobile environments. Procedia Comput. Sci. 2012, 10, 1202-1207.

86. Xamarin Inc. Xamarin. Available online: https://www.xamarin.com/platform (accessed on 29 January 2018).

87. Neyem, A.; Ochoa, S.F.; Pino, J.A.; Franco, R.D. A reusable structural design for mobile collaborative applications. J. Syst. Softw. 2012, 85, 511-524.

88. Armbrust, M.; Fox, A.; Griffith, R.; Joseph, A.D.; Katz, R.; Konwinski, A.; Lee, G.; Patterson, D.; Rabkin, A.; Stoica, I. A view of cloud computing. Commun. ACM 2010, 53, 50-58.

89. Ricci, F.; Rokach, L.; Shapira, B. Introduction to recommender systems handbook. In Recommender Systems Handbook; Springer: Boston, MA, USA, 2011; pp. 1-35.

90. Pazzani, M.J.; Billsus, D. Content-based recommendation systems. In The Adaptive Web; Springer: Berlin/Heidelberg, Germany, 2007; pp. 325-341.

91. Kim, D.; Yum, B.J. Collaborative filtering based on iterative principal component analysis. Expert Syst. Appl. 2005, 28, 823-830.

92. Allam, H.; Nassiri, N.; Rajan, A.; Ahmad, J. A critical overview of latest challenges and solutions of Mobile Cloud Computing. In Proceedings of the 2017 Second International Conference on Fog and Mobile Edge Computing (FMEC), Valencia, Spain, 8-11 May 2017; pp. 225-229.

93. Benedetto, J.I.; Valenzuela, G.; Sanabria, P.; Neyem, A.; Navón, J.; Poellabauer, C. MobiCOP: A Scalable and Reliable Mobile Code Offloading Solution. Wirel. Commun. Mob. Comput. 2018, 2018, 8715294.

(C) 2018 by the authors. Licensee MDPI, Basel, Switzerland. This article is an open access article distributed under the terms and conditions of the Creative Commons Attribution (CC BY) license (http://creativecommons.org/licenses/by/4.0/). 